\title{
Evoked Response Strength in Primary Auditory Cortex Predicts Performance in a Spectro-Spatial Discrimination Task in Rats
}

\author{
DElena Gronskaya and Wolfger von der Behrens \\ Institute of Neuroinformatics, University and ETH Zurich, 8057 Zürich, Switzerland
}

The extent to which the primary auditory cortex (A1) participates in instructing animal behavior remains debated. Although multiple studies have shown A1 activity to correlate with animals' perceptual judgments (Jaramillo and Zador, 2011; Bizley et al., 2013; Rodgers and DeWeese, 2014), others have found no relationship between A1 responses and reported auditory percepts (Lemus et al., 2009; Dong et al., 2011). To address this ambiguity, we performed chronic recordings of evoked local field potentials (eLFPs) in A1 of head-fixed female rats performing a two-alternative forced-choice auditory discrimination task. Rats were presented with two interleaved sequences of pure tones from opposite sides and had to indicate the side from which the higher-frequency target stimulus was played. Animal performance closely correlated $\left(r_{\mathrm{rm}}=0.68\right)$ with the difference between the target and distractor eLFP responses: the more the target response exceeded the distractor response, the better the animals were at identifying the side of the target frequency. Reducing the evoked response of either frequency through stimulus-specific adaptation affected performance in the expected way: target localization accuracy was degraded when the target frequency was adapted and improved when the distractor frequency was adapted. Target frequency eLFPs were stronger on hit trials than on error trials. Our results suggest that the degree to which one stimulus stands out over others within A1 activity may determine its perceptual saliency for the animals and accordingly bias their behavioral choices.

Key words: attention; auditory cortex; discrimination; evoked LFP; rats; SSA

Significance Statement

The brain must continuously calibrate the saliency of sensory percepts against their relevance to the current behavioral goal. The inability to ignore irrelevant distractors characterizes a spectrum of human attentional disorders. Meanwhile, the connection between the neural underpinnings of stimulus saliency and sensory decisions remains elusive. Here, we record local field potentials in the primary auditory cortex of rats engaged in auditory discrimination to investigate how the cortical representation of target and distractor stimuli impacts behavior. We find that the amplitude difference between target- and distractor-evoked activity predicts discrimination performance $\left(r_{\mathrm{rm}}=0.68\right)$. Specific adaptation of target or distractor shifts performance either below or above chance, respectively. It appears that recent auditory history profoundly influences stimulus saliency, biasing animals toward diametrically-opposed decisions.

\section{Introduction}

When it comes to stimulus processing functionality, describing the primary auditory cortex (A1) as "primary" may be somewhat misleading, as its role extends far beyond basic feature extrac-

\footnotetext{
Received March 29, 2018; revised April 19, 2019; accepted May 12, 2019.

Author contributions: E.G. and W.v.d.B. designed research; E.G. and W.v.d.B. performed research; W.v.d.B. contributed unpublished reagents/analytic tools; E.G. analyzed data; E.G. wrote the paper.

The authors declare no competing financial interests.

This work was supported by the Swiss National Science Foundation Grant Nr 172962. Wethank Kevan A. C. Martin for giving us the opportunity to develop a new direction of research in his laboratory, for investing his time and resources, and for encouraging and steering this work through many insightful discussions.

Correspondence should be addressed to Elena Gronskaya at elena@ini.ethz.ch.

https://doi.org/10.1523/JNEUROSCI.0041-18.2019

Copyright $\odot 2019$ the authors
}

tion (Nelken, 2004). Input to A1 already contains information about sound features such as frequency, interaural time, and level differences, and direction of spectral modulation (Wiegrebe and Winter, 2001). With a highly sparse stimulus representation (Hromádka et al., 2008; Rothschild et al., 2010), prominent responses to unpredictability (Näätänen et al., 1993; Tervaniemi et al., 1994; Bendixen et al., 2009), stimulus-specific adaptation (SSA; Ulanovsky et al., 2004) and rapid, behaviorally-driven receptive field plasticity (Fritz et al., 2003), A1 is considered to be the area where auditory scene analysis takes place. By extracting predictable patterns out of overlapping, multisource input, A1 is proposed to compute a dynamically-updated model of different sound sources in the 
environment, which are perceived as distinct "auditory objects" (Winkler et al., 2009).

Although the predictive coding properties of A1 have been established, the question of whether A1 activity reflects animals' sensory decisions remains debated. In A1 of cats performing a click rate discrimination task, no difference in activity between "go" and "no-go" trials was identified, leading the authors to conclude that A1 carries no information about the animals' judgment of the sound repetition rate (Dong et al., 2011). In ferret A1, however, activity changes were associated with the animals' judgment of pitch (Bizley et al., 2013). In monkeys, the evidence conflicts: whereas no decision-related activity was observed in A1 of animals engaged in an acoustic flutter discrimination task (Lemus et al., 2009), such changes were reported in monkeys discriminating amplitude modulation (Niwa et al., 2012) and frequency contours (Selezneva et al., 2006). In rat A1, meanwhile, evoked local field potential (eLFP) magnitude has been found to correlate with animal reaction times in a temporal expectation task (Jaramillo and Zador, 2011), whereas in animals alternating between sound discrimination and localization, prestimulus spike rates were shown to encode the stimulus selection rule (Rodgers and DeWeese, 2014).

To further explore the relationship between A1 activity and animal choice during auditory scene analysis, we trained rats in a two-alternative forced-choice (2-AFC) task that required them to distinguish two interleaved pure tone sequences of different frequencies and to report the location of the target sequence while ignoring the distractor. Because a given frequency could switch from functioning as the target to functioning as the distractor on a trial-by-trial basis, the rats had to flexibly modify their stimulus selection rule. A1 activity was recorded with chronicallyimplanted electrode arrays over many consecutive behavioral sessions. We found that the target/distractor frequency combinations that were the easiest for the rats to discriminate also showed the biggest amplitude differences in the population LFP responses that they evoked.

We went on to explore whether modifying that amplitude difference through SSA would affect animal performance in a predictable manner. As previously observed in the rat somatosensory cortex (Musall et al., 2014), adapted stimuli show weaker cortical responses and are more difficult for rats to detect than deviant stimuli. Conversely, stimuli that drive stronger population responses in mouse auditory cortex are behaviorally more salient (Deneux et al., 2016), and more salient auditory stimuli are easier to detect (Kayser et al., 2005). As expected, target localization performance improved when the distractor stimulus was adapted and deteriorated when the target stimulus was adapted. The difference in cortical eLFP response strength between the two competing stimuli closely correlated with animal performance. Moreover, the target response was on average stronger on trials in which animals responded correctly than on the error trials. These findings point toward the existence of a close link between A1 activity, stimulus saliency, and auditory choice in rats.

\section{Materials and Methods}

Animals and surgical procedures. All experimental and surgical procedures were approved by the Canton of Zürich Veterinary Office and conformed to the guidelines of the Swiss Animal Protection Law (Act of Animal Protection 16 December 2005 and Animal Protection Ordinance 23 April 2008). Six female adult Sprague-Dawley rats (rats 1-2 and 9-12; 260-430 g; Crl:SD, Charles River Laboratories) were trained in the behavioral task and electrophysiological recordings were obtained from three of the animals (rats 10-12; both hemispheres implanted). Animals were housed in groups of two in enriched-environment cages with ad libitum access to dry pellet chow and restricted access to water $5 \mathrm{~d}$ per week. When water-restricted, animals consumed their required daily water amount in the form of reward during morning and afternoon training sessions and also received supplementary water in the home cage at the end of the day. Animals were weighed daily to ensure that their weight did not decrease $<90 \%$ of their weekend baseline, when they had ad libitum water access. As nocturnal animals, the rats were kept under an inverted $12 \mathrm{~h}$ dark/light regimen, so that training and handling would fall into the active phase of their daily cycle.

Rats were implanted with headposts at $\sim 12$ weeks of age according to a previously-established protocol (Schwarz et al., 2010; Mayrhofer et al., 2013), with some modifications as described. The animal was anesthetized with isoflurane (1.8-2.5\% in oxygen; Attane, Piramal) and subcutaneously administered metazimole as analgesic (110 mg/kg body weight; Novaminsulfon, Sintetica), dexamethasone as an anti-inflammatory ( $3 \mathrm{mg} / \mathrm{kg}$ body weight, Helvepharm), and ceftriaxone as a preventative antibiotic (100 mg/kg body weight; Rocephin, Roche). Fur on the scalp was shaved and removed with depilatory cream (Veet), after which the animal was placed on a heating pad (Harvard Apparatus) controlled by feedback from a rectal probe to maintain body temperature at $37^{\circ} \mathrm{C}$. After application of vitamin A cream (Bausch \& Lomb) to the eyes, a surgical cloth was secured over the eyelids as counter-pressure to prevent isoflurane-induced edema. To prevent dehydration, warm isotonic glucose-electrolyte solution (Aequifusine; B. Braun Medical AG) was infused subcutaneously through an injection pump (Harvard Apparatus) at a rate of $2 \mathrm{ml} / \mathrm{h}$ throughout the duration of surgery.

The head was fixed in a stereotaxic frame (David Kopf Instruments) using blunt ear bars to avoid eardrum damage. The skull was exposed and cleaned, applying bupivacaine (Bucain, Actavis) as local anesthetic. Nine titanium screws (Modus 1.5, $3 \mathrm{~mm}$ length, Medartis) were inserted into the bone of the skull to provide anchors for the headcap at locations specified by Schwarz et al. (2010), omitting the two lateral screws above the temporal lobes. Following the application of a bonding agent (Prime \& Bond NT, Dentsply), the headcap was built up from layers of transparent light-curing dental cement (Tetric EvoFlow, Ivoclar Vivadent) polymerized with a handheld blue light source $\left(600 \mathrm{~mW} / \mathrm{cm}^{2}\right.$; Demetron). Black dental cement was used to mark the location of bregma and the center of Al for subsequent electrode implantation (5.2 mm caudal from bregma and $7 \mathrm{~mm}$ lateral from the midline; Szymanski et al., 2009). A screw (M5x15) was cemented into the headcap at the midline to create the headpost. The skin around the headpost was sutured and an antibiotic ointment was applied (Cicatrex, Janssen-Cilag). For recovery, animals were kept in cages placed on a heating pad and given enrofloxacin as antibiotic (200 mg/L drinking water; Baytril, Bayer) for 1 week postoperatively. Habituation to the head-fixation procedure was started only after complete healing of the skin around the headcap. To maintain the headcap in good condition, cleaning with $3 \%$ hydrogen peroxide was performed every $6-8$ weeks under anesthesia.

Following behavioral training, the rats underwent further surgery for the implantation of 4 -shank $\times 8$-channel linear probe arrays, tethered to an Omnetics head-stage connector (A4x8-5 mm-200-400-177-H32_21 $\mathrm{mm}$, impedances of $1-2 \mathrm{M} \Omega$ at $1 \mathrm{kHz}$; NeuroNexus). Animals were anesthetized and prepared for surgery as in the headpost-implantation procedure. Instead of a stereotaxic frame, a headpost holder was used to fix the head for drilling. The skull was thinned and a $\sim 2 \mathrm{~mm}^{2}$ craniotomy was made to expose the auditory cortex, after which a small slit in the dura was made to enable electrode insertion. Ringer's solution was used to protect the open brain during the implantation procedure. The ground and reference wires of the array were attached to one of the frontal headcap screws, which contacted the dura. The array was fastened to a custom-made guide pole, visually aligned for orthogonal cortex penetration, and advanced at $100 \mu \mathrm{m} / \mathrm{min}$ using a hydraulic drive (Trent Wells). When the tips of the probes were just inside the cortex, noise bursts and pure tones were presented to ensure that the region of insertion was responsive to the stimuli used in our paradigm and that the responses were characteristic of A1 (Profant et al., 2013). If no responses were obtained, the array was repositioned; otherwise, it was advanced 
until the top-most electrode channels were just visible at the cortical surface, allowing approximate depth estimation of the recording sites with respect to the cortical layers. The craniotomy was closed with silicone adhesive (Kwik-Cast, World Precision Instruments). Silver conductive paint (Electrolube) was applied over the grounding screws, wires, and the connector cable. The headcap was rebuilt with dental cement, leaving just the head-stage connector exposed, which was covered with a protective cap.

Electrophysiological recording. Recordings were performed from the day after the implantation for up to 3 months afterward, with spikes disappearing in 2-3 weeks to leave just the LFP. The recording signal was fed through a preamplifier ( $\mu$ PA32, $2 \times$ gain, Multi Channel Systems) and a signal collector (SC4x16-2x32 BC, Multi Channel Systems) into a 64-channel filter amplifier (FA64I, 600× gain, Multi Channel Systems), after which it was digitized at $32 \mathrm{kHz}$ and 12 bit on a PCI-bus data acquisition card (MC_Card, Multi Channel Systems). For all implanted arrays, neural tuning curves were determined in the first recording session after implantation in awake animals. Pure tones $(50 \mathrm{~ms}$, with $5 \mathrm{~ms}$ onset/offset cosine ramps) were presented from a speaker contralateral to the implanted hemisphere, sampling frequencies of $1-45 \mathrm{kHz}$ in 0.25 octave steps and sound pressure levels (SPLs) of $0-60 \mathrm{~dB}$ attenuation in $10 \mathrm{~dB}$ steps. Stimuli were presented for 20 repetitions in a pseudorandomized sequence at a rate of $1 \mathrm{~Hz}$. Tuning curves based on maximum multiunit spike counts in 5-15 ms bins within the first $0-100 \mathrm{~ms}$ of tone presentation were plotted in MATLAB 2011b (MathWorks). For sites with clear V-shaped tuning curves, characteristic frequencies (CFs; the frequency that evokes spikes at the lowest level) were visually estimated and classified as falling either below or above $16 \mathrm{kHz}$.

Implants were targeted to Al through stereotaxic coordinates $(5.2 \mathrm{~mm}$ caudal from bregma and $7 \mathrm{~mm}$ lateral from the midline) and stereotypical blood vessel patterns (Rutkowski et al., 2003; Kalatsky et al., 2005; Rutkowski and Weinberger, 2005; Polley et al., 2007). Array position was confirmed by identifying the strong, short-latency evoked responses typical for A1 (Profant et al., 2013). In addition, electrode tracks were verified histologically. Animals were deeply anesthetized, after which electrolytic lesions were made at the top and bottom recording sites of each shank and the animals were perfused transcardially with $4 \%$ paraformaldehyde in saline. The brain was sectioned into $80 \mu \mathrm{m}$ slices and Nissl stained to reconstruct the tracks. Because of the span of our arrays and unclear tuning of some units, we cannot exclude that some of our data comes from auditory fields of the core auditory cortex neighboring with A1.

Behavioral setup and stimuli. Behavioral training sessions were conducted in a sound-attenuated chamber, providing 50-60 dB attenuation at frequencies $>0.5 \mathrm{kHz}$ (A400, IAC Acoustics). Auditory stimuli were generated in MATLAB 2011b (MathWorks), converted to analog (NI PCI-4461, National Instruments), amplified (RB-1510 power amplifier, Rotel), and presented via a pair of speakers (R2904-700005 Tweeter, Scanspeak) placed $20 \mathrm{~cm}$ away from the animal's head at $45^{\circ}$ from the midline. To ensure a flat system transfer function within the stimulation frequency range, the speakers were calibrated (SigCalRP calibration software) using the RZ6 Multi I/O processor (Tucker-Davis Technologies) and microphone (model 4939; Brüel \& Kjær) positioned at the approximate location of the rat's ears. The head-fixation box was custom-built according to the design of Mayrhofer et al. (2013). Two water spouts, attached to piezo elements functioning as lick sensors (Bi-Morph, RS Components), were positioned in front of the animal's mouth. Water release was controlled by trigger-gated rocker solenoid valves (BürkertContromatic AG Schweiz). Two CCD finger-cameras (Conrad) with infrared illumination were installed above and below the animal's head to monitor behavior and stress levels. The behavioral apparatus was controlled via software custom-built in LabVIEW (National Instruments) interfacing with digital input/output hardware (NI PCIe-6321, National Instruments).

Behavioral training procedure and paradigms. In the first 2-4 weeks of training, animals were gradually familiarized with the experimenter, the behavioral set-up, and the head-fixation procedure in $10 \mathrm{~min}$ twice-daily handling sessions to minimize stress and facilitate cooperation and trust (Schwarz et al., 2010; Mayrhofer et al., 2013). The final 2-AFC paradigm
(Fig. 1) was shaped through intermediate stages of increasing difficulty over the course of another 8-12 weeks. As a first step, water-restricted rats learned to retrieve water from a central spout through operant conditioning: any lick of sufficient strength triggered valve opening to release $15-30 \mu \mathrm{l}$ of water. The valves made an audible click, providing a secondary source of reinforcement. Over the next few sessions, a second spout was introduced to teach animals to lick left and right.

Next, auditory localization training began. Trials were initiated automatically, with an intertrial interval of $1.5-2.5 \mathrm{~s}$, temporally jittered by up to $30 \%$. Stimuli consisted of $3 \mathrm{~s}$ sequences of pure tones or white noise bursts ( $50 \mathrm{~ms}$ long, with $5 \mathrm{~ms}$ on/off cosine ramps, $60 \mathrm{~dB} \mathrm{SPL}$ ), presented unilaterally at a repetition rate of $5 \mathrm{~Hz}$. Rats had to indicate the direction of sound (left or right) by licking the spout on the corresponding side. Importantly, the animals had to delay their response by $2 \mathrm{~s}$ from stimulation onset, giving a recording period free of motion artifacts. Opening of the response window was indicated by a $20 \mathrm{~dB}$ amplitude drop in the stimulus. Licks made outside of the response window or on the incorrect side were neither rewarded nor punished. For the first 20 training sessions ( $\sim 2000$ performed trials), the stimulus was presented from either the left or the right side in long blocks ( $>100$ trials). To prompt animals to switch from one side to the other, free reward was given from the appropriate spout for the first few trials of each new block. Blocks were progressively shortened until the animals readily switched sides trial-bytrial without prompting.

Frequency discrimination training was started once the animals' localization performance had reached $>70 \%$ and plateaued. Animals that were initially trained on white noise (rats 9-12) were first familiarized with pure tones on the localization task. Next, a distractor tone sequence, one octave below the target in frequency, was introduced from the opposite speaker with a temporal offset of $100 \mathrm{~ms}$, so that the target and distractor sequences were interleaved with no tone overlap at a joint repetition rate of $10 \mathrm{~Hz}$ (Fig. 1A). Two frequency pairings were used: $8 \mathrm{kHz}$ target $+4 \mathrm{kHz}$ distractor and $16 \mathrm{kHz}$ target $+8 \mathrm{kHz}$ distractor (8T4D and $16 \mathrm{~T} 8 \mathrm{D}$ shorthand). The frequency pairs were chosen so that all stimuli fell on a sensitive part of the rat's hearing curve (Kelly and Masterton, 1977) and so that there was sufficient spectral separation between them to promote stream segregation (Noda et al., 2013).

Initially, only one stimulus pair was presented for an entire training session, with the distractor at a decreased amplitude. Later in training, target and distractor were amplitude-matched, and the pairs were alternated first in blocks of trials and finally randomly trial-by-trial. Care was taken to expose the animals to both pairings evenly throughout the duration of training. As a final modification to the core paradigm, an adaptor sequence of either the target or the distractor frequency ( $50 \mathrm{~ms}$ pure tones at a $5 \mathrm{~Hz}$ repetition rate and $60 \mathrm{~dB} \mathrm{SPL}$ ) was presented bilaterally for $1 \mathrm{~s}$ before onset of the interleaved target-distractor sequence (in this case shortened from 3 to $2 \mathrm{~s}$ ). Adaptor frequencies were alternated either in trial blocks or on a trial-by-trial basis. Overall, there were six stimulus/ adaptation paradigm variations: $8 \mathrm{~T} 4 \mathrm{D}$ and $16 \mathrm{~T} 8 \mathrm{D}$ in the "No Adaptation", "Target Adapted", and "Distractor Adapted" conditions.

Experimental design and statistical analysis. All data analysis was performed in MATLAB 2011b (MathWorks). Electrophysiological recording data were obtained from three animals (6 hemispheres implanted with 32-channel arrays), providing over a hundred of independent recording sites. Meanwhile, behavioral data from six animals was analyzed to check that the performance of the three recorded subjects was representative of the larger group. Importantly, whenever a direct comparison was made between behavior and neural activity (see Figs. 2D, 3C, 4, 7), only those behavioral sessions in which the electrophysiological recordings were made were included in the dataset.

For behavioral analysis, trials were categorized into "hits", "errors", or "misses", depending on whether the animal licked the spout of the target side, the distractor side, or made no licks within the response window. Performance was calculated by dividing the total number of hits in each session by the sum of hits and errors. A cumulative Weibull function was fitted to performance over consecutive sessions of the localization paradigm (no distractor) to produce learning curves (Fig. $2 A$ ), with the dynamic learning phase defined as the range between the first and 
ninth decile of the function, as previously described by Mayrhofer et al. (2013). Behavioral sessions in which the animals performed very few trials $(<30)$ were excluded. Mean $(\mu) \pm$ SEM were used for reporting session trial counts. The Lilliefors test was used to verify a normal distribution.

Individual session performance distributions of the six different stimulus/adaptation paradigms were presented in notched box-and-whisker plots (see Figs. 3D, 4B). Box plot whiskers indicated maximum and minimum values; edges: the 25th (q1) and 75th (q3) percentiles; central line: the median (M); notches: the $95 \%$ confidence intervals (CIs) around M, with notch extremes calculated as $\mathrm{M} \pm 1.57(\mathrm{q} 3-\mathrm{q} 1) / \operatorname{sqrt}(n)$, where $n$ is the number of sessions. The Wilcoxon rank sum test was used to compare performance of the $8 \mathrm{~T} 4 \mathrm{D}$ versus the $16 \mathrm{~T} 8 \mathrm{D}$ stimulus pairs, while the Wilcoxon signed rank test was used to compare performance of the target-adapted versus distractor-adapted conditions for each stimulus pair. The Kruskal-Wallis test was used to determine whether stimulus frequency affected animal performance in either localization or discrimination. If so, Bonferroni-corrected post hoc pairwise comparisons were performed at a $p<0.05$ minimal significance level. For illustration of intersubject performance variability (Fig. $2 B$ ), trials across all sessions of particular paradigm type were taken and $95 \%$ binomial confidence intervals were computed.

Electrophysiological recording data were preprocessed with MC_Rack software v4.1.1 (Multi Channel Systems) to cut the session-long recordings into single-trial segments. In implants with deficient grounding (rat10, right hemisphere; rat11, right hemisphere; rat12, left hemisphere), MC_Rack was used to re-reference all channels to a recording channel at the cortical surface. Data from one of the arrays was excluded in the No Adaptation paradigm (rat11, right hemisphere) and from another in the Target Adapted and Distractor Adapted paradigms (rat10, left hemisphere), because of high levels of $50 \mathrm{~Hz}$ noise and/or too few sessions of that particular paradigm type. Given that cells in A1 are primarily driven by contralateral stimuli (Yao et al., 2013), only responses to tones presented from the side contralateral to the recorded hemisphere were considered.

Raw recording data were bandpass filtered (Butterworth second order, $500 \mathrm{~Hz}-5 \mathrm{kHz}$ ) and spikes were identified as negative peaks exceeding 5 SD of the signal waveform (but not $>20$, to exclude movement artifacts). Peristimulus time histograms (PSTHs) were calculated from multiunit activity using $5 \mathrm{~ms}$ bins. Local field potentials were obtained by low-pass filtering (Butterworth 4 th order, $1 \mathrm{kHz}$ ) and resampling the raw data at 1 $\mathrm{kHz}$. Waveforms with absolute values exceeding $3 \mathrm{mV}$ were excluded as movement artifacts.

Given that the 32-channel arrays sampled activity every $200 \mu \mathrm{m}$ vertically, across all cortical layers, and every $400 \mu \mathrm{m}$ horizontally across 1.2 $\mathrm{mm}$ of cortical surface, a population overview of the target- and distractor-driven activity in A1 could be obtained. First, single-trial LFP waveforms were averaged across all trials of each recording session for each channel. From the mean waveform, the absolute value of the negative inflection peak detected within $10-25 \mathrm{~ms}$ of sound onset was taken as the tone-evoked response amplitude. If the waveform peak confidence intervals overlapped in consecutive recording sessions, the recording was considered stable and the responses were not taken as independent data points but were pooled and averaged. Only responses to the first target and distractor tones of the interleaved sequence were analyzed. Values above the 95th percentile and below the fifth percentile of the dataset were excluded as outliers. The resulting distributions of the target and distractor eLFP amplitudes were presented as box-and-whisker plots (see Figs. 3C, $4 A$ ) and compared in different stimulus and adaptation conditions using the Wilcoxon rank sum test or the Wilcoxon signed rank test, as appropriate.

To gauge whether a given recording site was more responsive to the target or the distractor, an index of target predominance was calculated for each channel in each recording session $\left[T_{\mathrm{idx}}=\left(\mathrm{eLFP}_{\mathrm{targ}}-\mathrm{eLFP}_{\mathrm{dist}}\right) /\right.$ $\left.\left(\mathrm{eLFP}_{\text {targ }}+\mathrm{eLFP}_{\text {dist }}\right)\right]$, where eLFP is the mean response amplitude evoked by the first target or distractor tone in the interleaved sequence, as described above). At least one of the responses used to calculate $T_{\mathrm{idx}}$ had to fall above the threshold of $50 \mu \mathrm{V}$ (with reference to the LFP waveform) in order for the recording site to be considered. The other responses, if below threshold, were defined as the minimum value of the LFP waveform within 10-25 ms of sound onset. For the $T_{\mathrm{idx}}$ distributions in Figure 6 , responses in each session were analyzed separately for hit, error, and miss trials. In Figure 7, median $T_{\text {idx }}$ values were calculated for each implanted array, pooling all sessions and behavioral response categories (in $A$ ) or all trials in a single session (in $B$ ). Median animal performance in the behavioral session(s) during which that array's recordings were made $\left(M_{\text {perf }}\right)$ was plotted as a function of $T_{\mathrm{idx}}$ for the six different paradigms. A linear regression model was fitted to the data (MATLAB function "fitlm"; Fig. 7A). Repeated measures correlation (rmcorr), a statistical method developed for datasets in which multiple measurements are performed per subject (Bakdash and Marusich, 2017), was used to assess the intraindividual association between $T_{\mathrm{idx}}$ and behavioral performance that all subjects share (Fig. 7B). Rstudio v1.1.463 (https://www.rstudio.com/) was used to implement the "rmcorr" package.

\section{Results}

A novel spectra-spatial 2-AFC auditory discrimination task in head-fixed rats

We established a new behavioral paradigm of auditory scene analysis for rats that models the "cocktail party problem" (Cherry, 1953), a situation in which one must identify and respond to a behaviorally-relevant auditory "target" in the presence of qualitatively-similar "distractors". In our paradigm (Fig. 1), the competing stimuli consisted of trains of pure tones, the target stimulus one octave higher in frequency than the distractor. Two frequency pairs were used: $8 \mathrm{kHz}$ target $+4 \mathrm{kHz}$ distractor (8T4D) and $16 \mathrm{kHz}$ target $+8 \mathrm{kHz}$ distractor (16T8D). These were alternated within a single behavioral session either in blocks or trial-by-trial. Because $8 \mathrm{kHz}$ switched roles from target to distractor depending on frequency pairing, rats had to flexibly adjust their stimulus selection rule.

The monaural target and distractor tone sequences were presented from opposite speakers, with presentation sides switching randomly trial-by-trial. The animals, head-fixed, had to report the speaker from which the target stimulus was presented (left or right) by licking a water spout on the corresponding side. Although the target frequency could, in principle, be identified as soon as the first tones of the competing stimuli were heard and compared, the window in which a lick response would trigger release of reward opened only $2 \mathrm{~s}$ after stimulus onset, which was signaled by a $20 \mathrm{~dB}$ SPL amplitude drop in the target (Fig. 1A). Rats quickly learned to time their licks to the amplitude drop, providing an extensive recording period free of movement artifacts. As a modification to the core task, a binaural adaptor sequence of either the target or the distractor frequency was presented for the first second of each trial (Fig. $1 B$, paradigms ii and iii).

Shaping of the final paradigm through simpler stages took 8-12 weeks. The critical shaping step that took the longest for the animals to master was sound localization (without distractor) in the 2-AFC setting: responding left or right according to the direction of sound. Learning curves for the acquisition of this behavior (Fig. 2A) reveal variability of the individual animals in the dynamic learning phase, the part of the learning curve where $80 \%$ of the change in performance occurs. When pure tones exclusively were used in localization training, the animals' dynamic learning phase was 1905 trials (rat1) and 395 trials (rat2), though it is interesting to note that the slower animal ultimately reached a better stable performance than the faster animal [mean and $95 \%$ binomial CIs (CI95) for rat1: 83.5\% (82.0-85.0\% CI95); for rat2: $77.7 \%(75.8-79.5 \%$ CI95)]. When white noise was used in the initial training to facilitate localization as a more spectrally-rich stimulus, the dynamic learning phase remained in a similar 


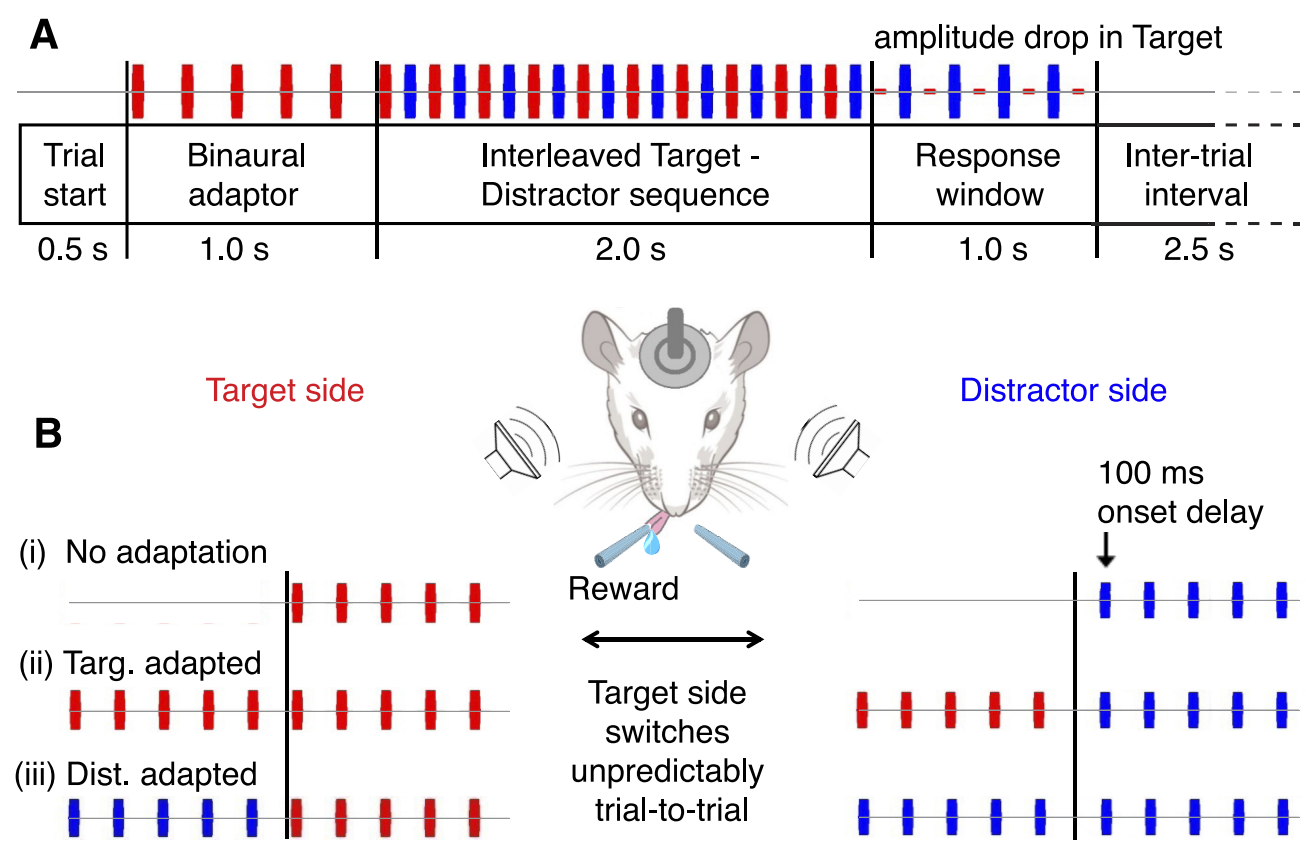

Figure 1. The 2-AFC spectra-spatial auditory discrimination task. $A$, Trial timing schematic. At the start of the trial, the rat hears an adaptor sequence followed by an interleaved target-distractor sequence of high-low pure tones (target, red; distractor, blue; 50 ms tone duration; $10 \mathrm{~Hz}$ joint repetition rate; $60 \mathrm{~dB}$ SPL amplitude). A $20 \mathrm{~dB}$ SPL amplitude drop in the target signals the opening of the $1 \mathrm{~s}$ response window. Early responses are neither rewarded nor punished. $\boldsymbol{B}$, Stimulus configuration by paradigm type. In paradigm (i), no adaptor is present. The first stimulus heard by the rat is the target tone (played from either the left or the right speaker with random assignment on every trial), which is followed by the distractor tone from the opposite speaker with a 100 ms delay, resulting in an interleaved high-low tone sequence. The rat has to localize the target stimulus (the higher frequency of the pair) and to respond with a lick on the target side. In the adaptation paradigms, five tones of either the target frequency (ii) or the distractor frequency (iii) are presented simultaneously from both speakers for the first second of the trial before the onset of the interleaved sequence. The Target adapted paradigm (ii) is shown in $(\boldsymbol{A})$.

range: 928 trials (rat9), 1372 trials (rat10), 999 trials (rat11), and 738 trials (rat12), as did the plateau performance: $87.9 \%(86.4-$ $89.4 \%$ CI95; rat9), $85.4 \%$ (83.4-87.3\% CI95; rat10), $80.1 \%$ (78.6-81.6\% CI95; rat11), and 86.3\% (84.4-88.1\% CI95; rat 12$)$. Upon transitioning to pure tone localization, performance of two of the animals fell [64.0\% (50.0-76.0\% CI95) first pure tone session performance for rat10 and 62.7\% (53.6-71.8\% CI95) for rat12], recovering only partially in the subsequent sessions. Importantly, all four of the animals that were initially trained on white noise successfully generalized to pure tones, reaching $>70 \%$ correct already on the second behavioral session after the transition.

Although only $8 \mathrm{kHz}$ localization data are shown for rats 9-12 in Figure $2 A$ for comparability to the behavior of rat1 and rat2, the animals were trained and tested on a dozen of additional pure tone frequencies in the $1-40 \mathrm{kHz}$ range to assess the dependence of localization performance on frequency and to avoid overexposure to $8 \mathrm{kHz}$. Rats performed equally well at localizing white noise, 8 and $16 \mathrm{kHz}$, but $4 \mathrm{kHz}$ performance was significantly lower (Fig. $2 B ; p<10^{-9}$, Kruskal-Wallis test with post hoc pairwise Wilcoxon rank sum tests, Bonferroni-corrected for multiple comparisons). This finding was not considered a concern for interpretation of performance on the final discrimination paradigm, given that $4 \mathrm{kHz}$ functioned as a distractor and its localization was never required.

Training of spectra-spatial discrimination was started once localization performance had reached a plateau. The distractor stimulus was introduced at a lower amplitude (10-20 dB SPL), which was increased to match the target amplitude over the next few training sessions. Animal performance was barely affected by the introduction of a lower-volume distractor, dropped as distractor volume was raised, and recovered over the next several sessions to a new stable performance (Figs. 2C, 3D). All of the animals trained succeeded in learning the final paradigm. Rats performed an average of 240 trials per hour-long session twice daily, the best session counts topping 700 trials (mean trial count \pm SEM for rat1: $103 \pm 16$, rat2: $184 \pm 8$, rat9: $346 \pm 20$, rat10: $238 \pm 9$, rat11: $419 \pm 12$, rat12: $153 \pm 5$ ).

\section{Encoding of pure tone sequences in rat A1}

LFPs and multiunit activity (MUA) were recorded using 4-shank-by-8-channel silicon probe arrays bilaterally implanted in A1 of three of the six trained animals (rats 10-12). Given the complementary lateralization of the spatial receptive fields in rat auditory cortex, we did not see a need to distinguish between the hemispheres for this type of task, particularly because very little difference in the activity of left and right auditory cortex was recently reported in monkeys performing a similar paradigm of pure tone categorization (Selezneva et al., 2017). Pure tones evoked sharp, short-latency negative current responses in the LFP, which adapted to slightly lower amplitude for the subsequent tones in the sequence (Fig. $3 A$, left). Adaptation was more pronounced in the spiking responses (Fig. $3 A$, right). As previously shown by Gaucher et al. (2012), LFPs were more broadly tuned than MUA: tones could evoke strong negative inflections in the LFP at some recording sites without driving spikes (Fig. $3 A$, compare LFP and MUA of channel 15 for 8T4D and channel 7 for 16T8D). Strongest LFP responses were evoked $400-800 \mu \mathrm{m}$ below the cortical surface (Fig. $3 B$ ), corresponding to the thalamocortical input layer IV (Szymanski et al., 2009).

In Sprague-Dawley rats, A1 extends for 3-4 $\mathrm{mm}$ across the cortex, neuronal CFs running from $<1 \mathrm{kHz}$ at the caudal-most boundary to $50 \mathrm{kHz}$ at the rostral-most boundary (Sally and Kelly, 1988; Rutkowski et al., 2003). Spanning $1.2 \mathrm{~mm}$ of A1 in 

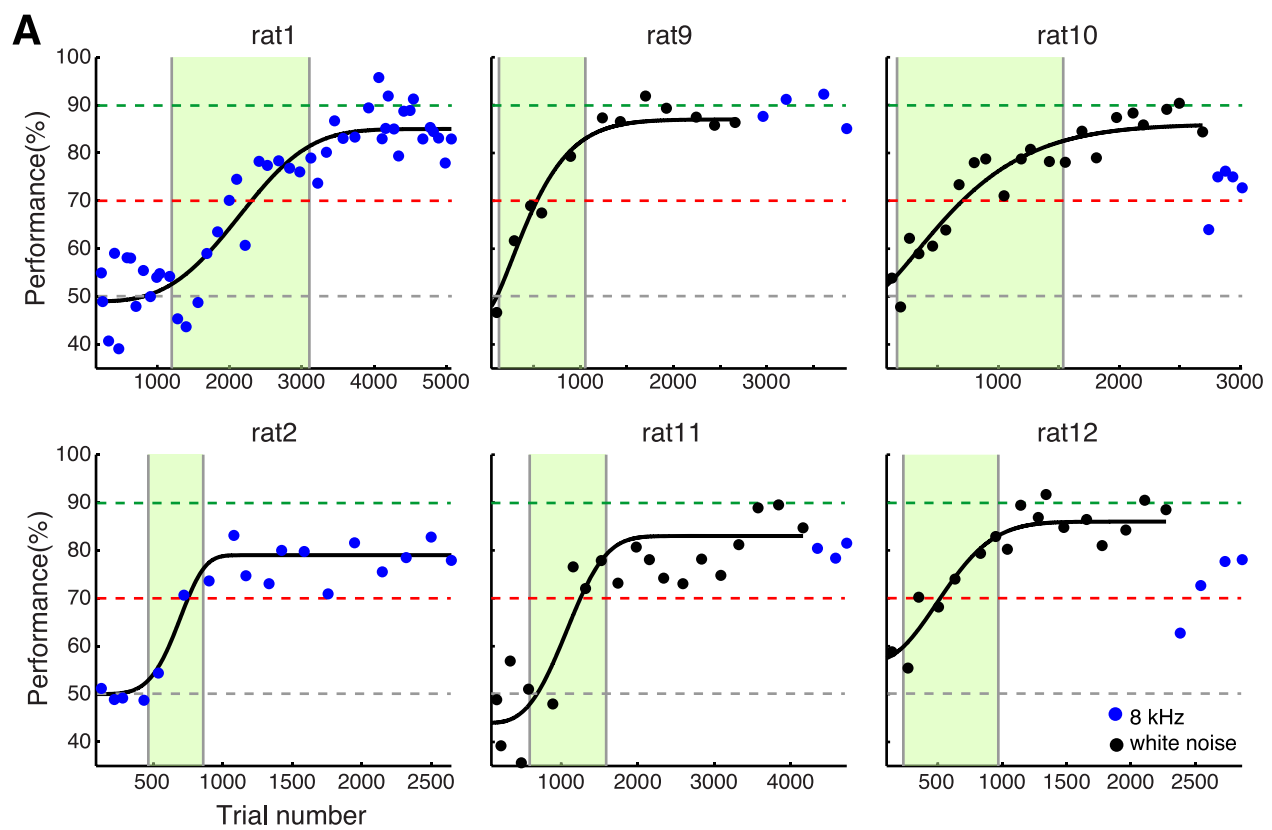

\section{B}

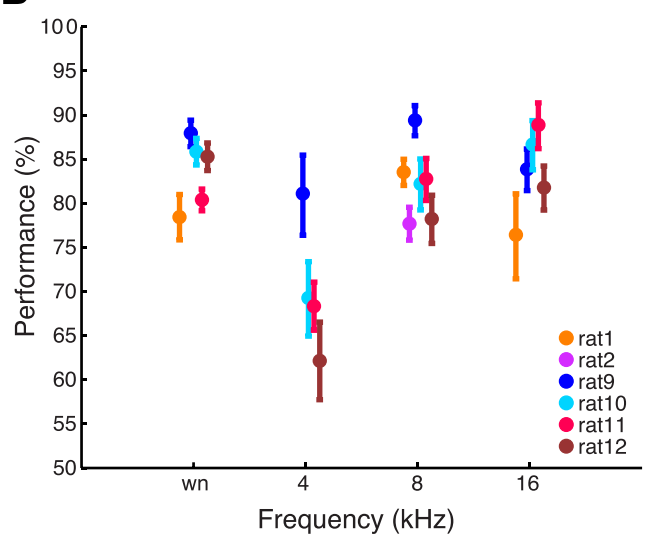

C

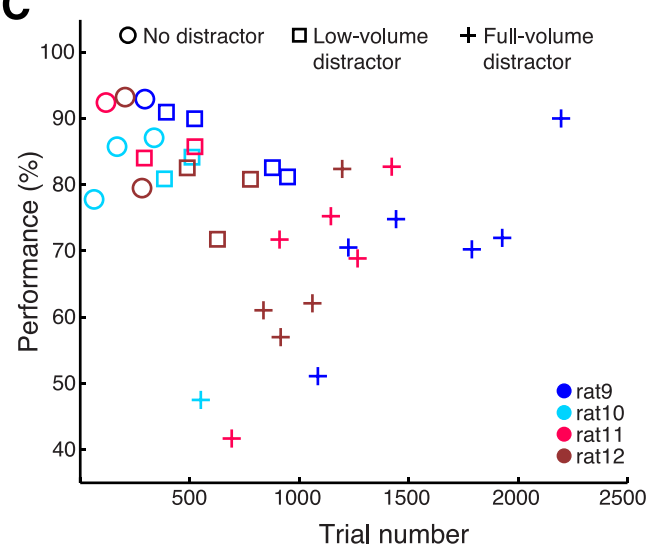

Figure 2. Individual animal task acquisition through consecutive shaping stages. $A$, Learning curves for sound localization without distractor. Rat 1 and rat2 2 were trained on pure tones $(8 \mathrm{kHz}$ tone sequence; blue marker), whereas rats $9-12$ were trained on white noise (same duration and amplitude parameters; black marker) and transitioned to pure tones once stable performance was reached. A cumulative Weibull function, fitted to the mean performance of consecutive behavioral sessions, shows each animal's dynamic learning phase (shaded green). $\boldsymbol{B}$, Stable localization performance for white noise (wn), 4, 8, and $16 \mathrm{kHz}$ (the pure tones used in the final spectra-spatial discrimination task). Mean performance and 95\% binomial Cls are shown. C, Individual performance for rats 9 -12 during the transition from the localization paradigm (no distractor) to the final target-distractor discrimination paradigm. Mean session performance is shown for $16 \mathrm{kHz}$ localization without distractor (circles), with distractor at a $10-20 \mathrm{~dB}$ lower amplitude (squares), and finally with distractor amplitude-matched to the target (crosses; $60 \mathrm{~dB} \mathrm{SPL).} \mathrm{Performance} \mathrm{falls}$ only when the full-volume distractor is introduced and quickly recovers. Behavioral sessions with the $8 \mathrm{kHz}$ target were interspersed in the training (data not shown for clarity). Rat1 and rat2 were transitioned to the discrimination paradigm using a different pure tone frequency $(24 \mathrm{kHz})$, thus data are not shown.

the rostral-caudal direction, our arrays covered at least two octaves of CFs on the tonotopic map. Of all our recorded multiunits with stereotypical V-shaped tuning curves, a majority had CFs $>16 \mathrm{kHz}$ (78/126 MUs), consistent with others' finding that higher frequencies are over-represented in the rat auditory cortex, with the upper three octaves of the rat's hearing range taking up $\sim 80 \%$ of space in acoustically-responsive areas (Sally and Kelly, 1988).

\section{Eight kilohertz drives the strongest population response in} rat $\mathrm{A} 1$ and rats perform best when $8 \mathrm{kHz}$ is the target Figure $3 C$ shows the A1 population distributions of eLFP response amplitudes evoked by the first target tone and the first distractor tone of the interleaved sequence, recorded while the animals performed the No Adaptation paradigm. We restricted our analysis to responses driven by the first two tones in the interleaved sequence, which carry all the information necessary for rats to compare the frequencies and identify the side of the target. At $60 \mathrm{~dB}$ SPL, the strongest responses were driven by 8 $\mathrm{kHz}$, whether it was the target in the 8T4D pairing $(\mathrm{M} \pm 95 \% \mathrm{CI}$ for $8 \mathrm{kHz}: 154 \pm 13 \mu \mathrm{V}$ and for $4 \mathrm{kHz}: 72 \pm 7.6 \mu \mathrm{V} ; n=241, p<$ $10^{-27}$, Wilcoxon signed rank test) or the distractor in the 16T8D pairing $(\mathrm{M} \pm 95 \% \mathrm{CI}$ for $8 \mathrm{kHz}: 115 \pm 12 \mu \mathrm{V}$ and for $16 \mathrm{kHz}$ : $83 \pm 8.6 \mu \mathrm{V} ; n=238, p<10^{-15}$, Wilcoxon signed rank test). The response to $8 \mathrm{kHz}$ was higher when it was the target than when it was the distractor $\left(p<10^{-5}\right.$, Wilcoxon rank sum test), presumably because the distractor was always presented $100 \mathrm{~ms}$ after the target and was therefore subject to a degree of nonspecific adaptation.

As shown in Figure 3D, rats performed much better when 8 $\mathrm{kHz}$ was the target rather than the distractor stimulus: target localization accuracy was $78 \pm 3.5 \%$ for $8 \mathrm{~T} 4 \mathrm{D}$ but only $54 \pm$ 

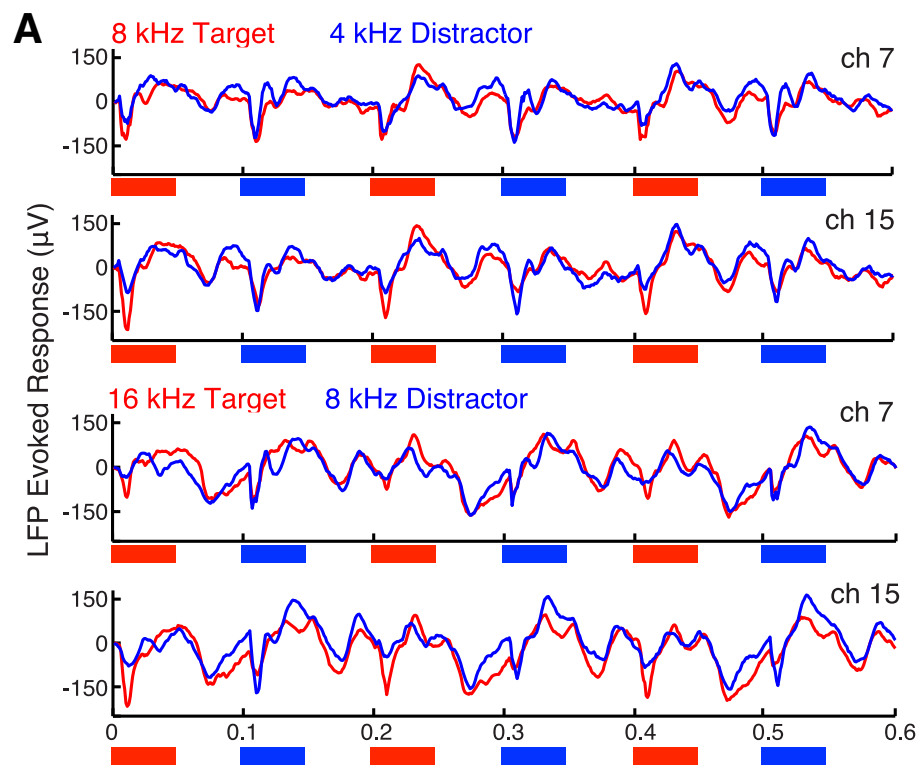

B

Time (s)

Cortical depth $(\mu \mathrm{m})$ :

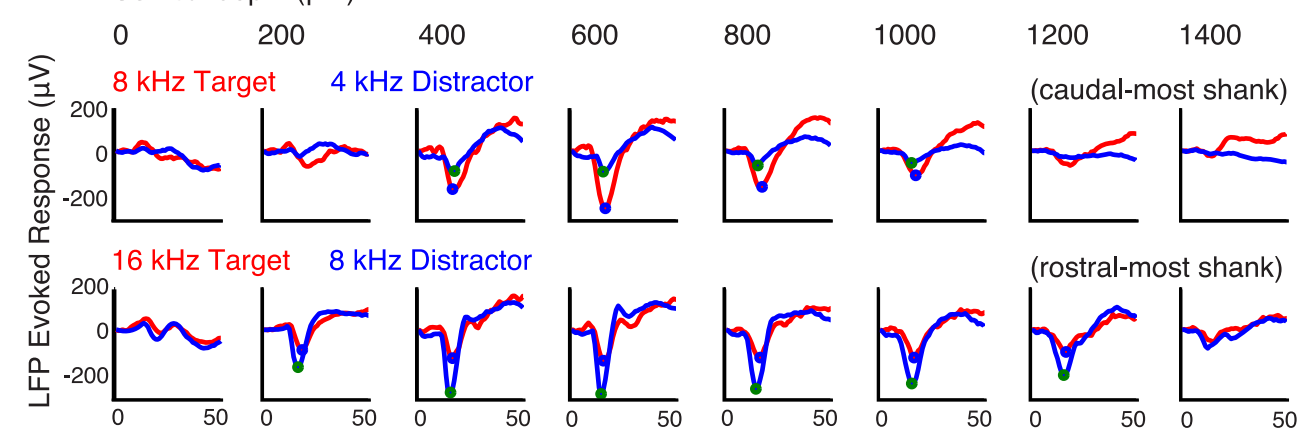

Time (ms)

C

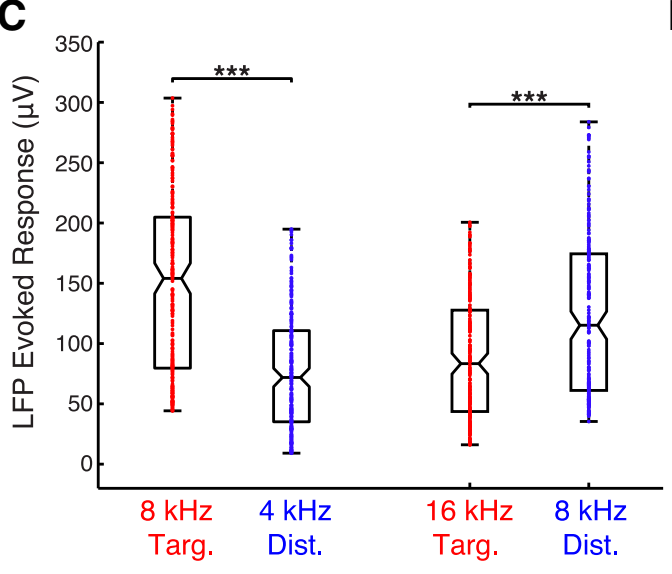

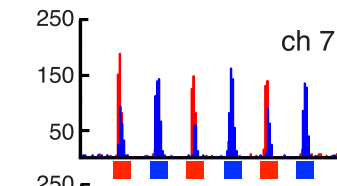

$\operatorname{ch} 15$

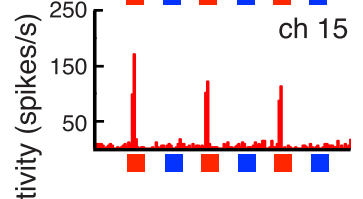

$\operatorname{ch} 7$
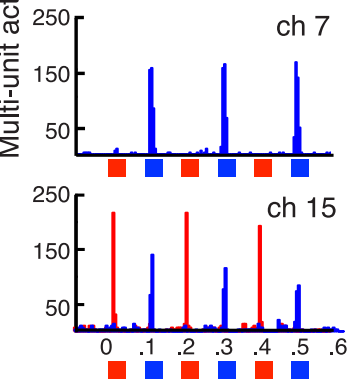

Time (s)

1400

D

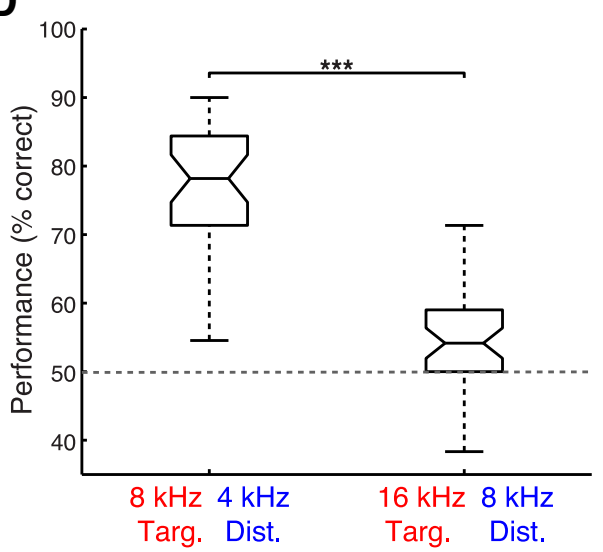

Figure 3. Neural responses to pure tone sequences in rat A1 and their discriminability in the 2-AFC task. A, Mean LFP traces and corresponding MUA PSTHs for two channels on neighboring shanks in an example recording session of a passively-listening animal. Evoked responses to the first six tones of the interleaved target/distractor sequence (8T4D, top; 16T8D, bottom) are shown. Red traces and red PSTH bars (5 ms bins) correspond to trials in which the target was presented contralaterally to the recorded hemisphere; blue, to trials in which the distractor was presented contralaterally. $B$, Cortical depth profile for tone-evoked LFP responses on a caudal-most and a rostral-most shank in an example recording session of a behaving animal. eLFP responses to the first target and first distractor tones of the interleaved sequence are shown overlaid (8T4D, top; 16T8D, bottom). Though the shanks are separated by $1200 \mu \mathrm{m}$ on the auditory cortical tonotopic map, $8 \mathrm{kHz}$ drives the stronger response in both locations. $\boldsymbol{C}$, Absolute values of the first-tone eLFP waveform minima ( $\boldsymbol{B}$, circled blue and green) are taken from all the tone-responsive channels of five array implants in three behaving animals over 56 recording sessions to show the population distributions of eLFP amplitudes evoked by the target and distractor tones in rat A1. The median response for $8 \mathrm{kHz}$ is stronger whether it is the target in the $4 \mathrm{kHz}$ pairing (left box plots; $n=241$ ) or the distractor in the $16 \mathrm{kHz}$ pairing (right box plots; $n=238 ;$ Wilcoxon signed rank test, ${ }^{* * *} p<0.001$ ). D, Distribution of mean animal performance in individual behavioral sessions for $8 \mathrm{~T} 4 \mathrm{D}$ (left; 35 sessions) and 16T8D (right; 41 sessions). Rats perform better when $8 \mathrm{kHz}$ is the target rather than the distractor (Wilcoxon rank sum test, ${ }^{* * *} p<0.001$ ). 

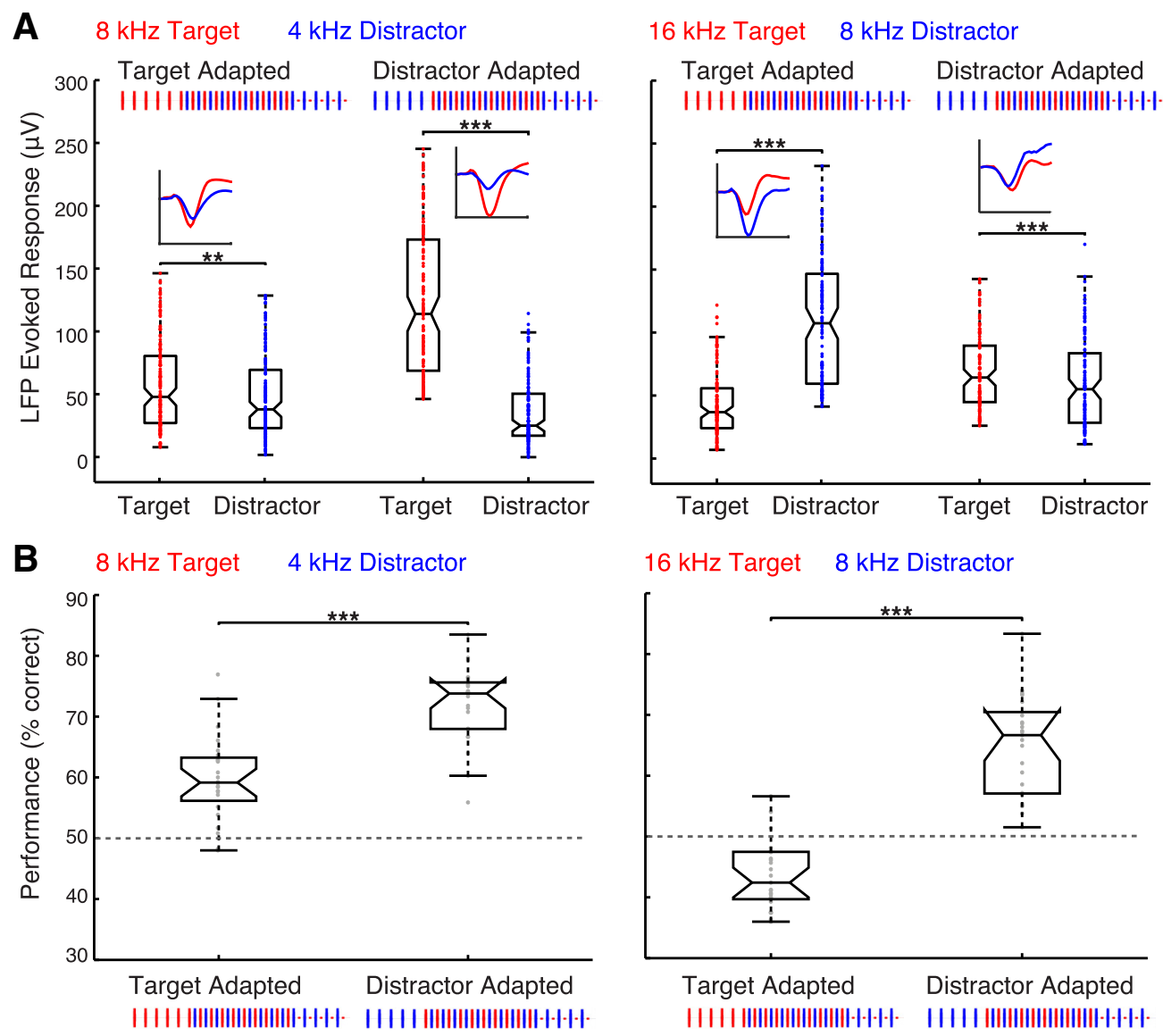

$16 \mathrm{kHz}$ Target $8 \mathrm{kHz}$ Distractor

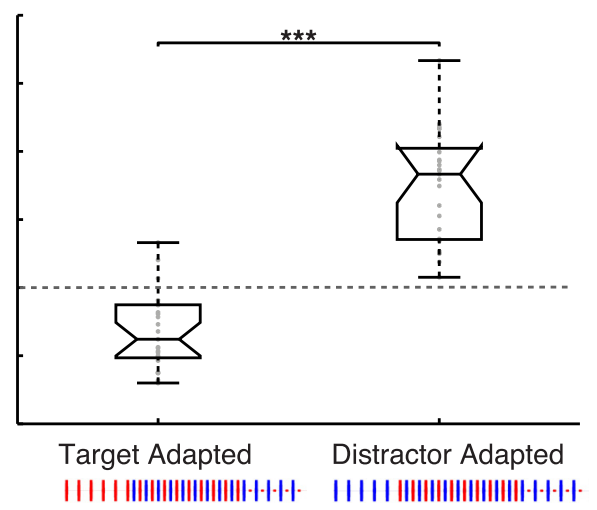

Figure 4. Biasing auditory cortical responses in favor of the target through stimulus-specific adaptation improves animal performance. A, Stimulus-specific adaptation in the eLFP population response of A1 for 8T4D (left) and 16T8D (right). Insets, Mean waveforms, averaged over all recording sessions and channels for a representative implant (rat12; left hemisphere; 7 recording sessions), of eLFP responses driven by the first target and the first distractor tone following the binaural adaptor sequence. Waveforms are overlaid to illustrate how the target and distractor eLFP amplitudes change with respect to each other in the different adaptation conditions. Adapting the target favors the distractor response; adapting the distractor favors the target response. Box plots show eLFP response amplitude distributions in behaving animals (31 recording sessions in 3 rats). Each data point corresponds to the mean eLFP response of one channel in one recording session (or several consecutive sessions; see Materials and Methods). Differences in population response amplitudes for the target and distractor are significant in all stimulus/adaptation configurations (Wilcoxon signed rank test; ${ }^{* *} p<0.01,{ }^{* * *} p<0.001 ; n=150$ for 8T4D Target Adapted; $n=141$ for 8T4D Distractor Adapted; $n=126$ for 16T8D Target Adapted; $n=121$ for 16T8D Distractor Adapted). $\boldsymbol{B}$, Distributions of mean animal performance in individual sessions for 8T4D (left; 24 recording sessions) and 16T8D (right; 25 recording sessions). Just as the target eLFP response is strongest when the distractor is adapted, animal performance is best when the distractor is adapted (right box plots in both panels; Wilcoxon signed rank test, ${ }^{* * *} p<0.001$ ).

$2.2 \%$ for $16 \mathrm{~T} 8 \mathrm{D}(\mathrm{M} \pm 95 \% \mathrm{CI}, n=35$ and $n=41$ sessions, respectively; $p<10^{-12}$, Wilcoxon rank sum test). A possible connection between A1 activity and animal behavior emerges. When the population evoked response to the target stimulus is greater than the response to the distractor, the animals distinguish and localize the target at high accuracy, but when the response to the distractor is greater, performance drops to near-chance.

Behavioral performance can be improved or degraded by reducing the evoked response to the distractor or the target, respectively, through stimulus-specific adaptation

Having observed a link between animal performance and the relative amplitude of target and distractor evoked responses, we went on to ask whether amplifying or reducing this difference would influence behavior in the expected direction. Our prediction was the following: the more the A1 population response to the target exceeds the distractor response, the more likely the animal will be to respond on the side of the target stimulus. Conversely, the stronger the distractor response is in relation to the target, the more mistakes the animal will make by selecting the side of the distractor stimulus.
One approach for changing the amplitude of auditory cortical responses in a stimulus-targeted manner is to present an adapting sequence first. As established in previous studies of the effects of stimulus probability on neural response amplitude (Ulanovsky et al., 2004; von der Behrens et al., 2009), rare deviant tones interspersed within a sequence of standards drive stronger responses than the frequent standards. We made use of this "oddball effect" to design a modified version of our task. For the first second of each trial, before the onset of the interleaved target/distractor sequence, we presented a binaural adaptor sequence of either the target or the distractor frequency (Fig. $1 B$, paradigms ii and iii). We predicted that pre-adapting the distractor, thereby making the target the deviant, would enhance the evoked response to the target and improve behavioral performance, whereas preadapting the target to make the distractor the deviant would have the opposite effect, degrading performance.

The effects of adaptation and deviance on the eLFP amplitudes are apparent when comparing the Target Adapted and the Distractor Adapted distributions in Figure 4A. For 8T4D, the evoked response to $8 \mathrm{kHz}$ is much lower in the target-adapted than in the distractor-adapted condition (Wilcoxon rank sum test, $p<10^{-22}$ ), whereas the $4 \mathrm{kHz}$ response is lower in the 
distractor-adapted than in the target-adapted condition (Wilcoxon rank sum test, $p<10^{-4}$ ). Similarly, for $16 \mathrm{~T} 8 \mathrm{D}$, the response to $16 \mathrm{kHz}$ is lower in the target-adapted condition (Wilcoxon rank sum test, $p<10^{-12}$ ), whereas the $8 \mathrm{kHz}$ response is lower in the distractor-adapted condition (Wilcoxon rank sum test, $p<10^{-14}$ ).

Same as in the No Adaptation paradigm (Fig. $3 C$ ), $8 \mathrm{kHz}$ evoked a weaker response as the distractor in the $16 \mathrm{~T} 8 \mathrm{D}$ pairing than as the target in the $8 \mathrm{~T} 4 \mathrm{D}$ pairing $(p=0.04$, Wilcoxon rank sum test), though this difference was only significant when $8 \mathrm{kHz}$ was not adapted in a frequency-specific manner (Fig. 4A; 8T4D, 4 $\mathrm{kHz}$ adapted vs $16 \mathrm{~T} 8 \mathrm{D}, 16 \mathrm{kHz}$ adapted). The strong reduction of the $8 \mathrm{kHz}$ response by a preceding $8 \mathrm{kHz}$ sequence apparently masked any nonspecific adaptation effects (Fig. 4A; 8T4D, $8 \mathrm{kHz}$ adapted vs $16 \mathrm{~T} 8 \mathrm{D}, 8 \mathrm{kHz}$ adapted; $p=0.7$, Wilcoxon rank sum test). Curiously, the stimuli giving the weaker population responses (16 and $4 \mathrm{kHz}$ ) also showed a much smaller effect of stimulus-specific adaptation [34\% reduction in the median evoked response for $4 \mathrm{kHz}$ compared with $58 \%$ reduction for 8 $\mathrm{kHz}$ (Fig. $4 B$, left) and $42 \%$ reduction for $16 \mathrm{kHz}$ compared with $49 \%$ reduction for $8 \mathrm{kHz}$ (Fig. $4 B$, right)]. These results imply that stimuli strongly represented in auditory cortex have the widest dynamic range for adaptation.

Overall, in three out of the four stimulus/adaptation configurations, the stronger population response was driven by $8 \mathrm{kHz}$, just as in the No Adaptation paradigm. For 8T4D (Fig. 4A, left), amplitude of $8 \mathrm{kHz}$ greatly exceeded $4 \mathrm{kHz}$ in the distractoradapted condition $(\mathrm{M} \pm 95 \% \mathrm{CI}$ for $8 \mathrm{kHz}$ : $114 \pm 14 \mu \mathrm{V}$ and for $4 \mathrm{kHz}: 25 \pm 4.4 \mu \mathrm{V} ; n=141, p<10^{-25}$, Wilcoxon signed rank test) as well as in the target-adapted condition, albeit to a smaller extent $(\mathrm{M} \pm 95 \% \mathrm{CI}$ for $8 \mathrm{kHz}$ : $48 \pm 6.8 \mu \mathrm{V}$ and for $4 \mathrm{kHz}: 38 \pm$ $5.9 \mu \mathrm{V} ; n=150, p=0.008$, Wilcoxon signed rank test). Similarly, the $8 \mathrm{kHz}$ response was overall higher in the 16T8D Target Adapted paradigm $(\mathrm{M} \pm 95 \% \mathrm{CI}$ for $8 \mathrm{kHz}: 107 \pm 12 \mu \mathrm{V}$ and for $16 \mathrm{kHz}: 37 \pm 4.4 \mu \mathrm{V} ; n=126, p<10^{-20}$, Wilcoxon signed rank test). When adapted, however, the $8 \mathrm{kHz}$ response was reduced sufficiently for the $16 \mathrm{kHz}$ target to become stronger $(\mathrm{M} \pm 95 \%$ $\mathrm{CI}$ for $8 \mathrm{kHz}$ : $55 \pm 7.9 \mu \mathrm{V}$ and for $16 \mathrm{kHz}$ : $64 \pm 6.4 \mu \mathrm{V} ; n=121$, $p<10^{-4}$, Wilcoxon signed rank test). Importantly, this was the only stimulus/adaptation configuration in which $8 \mathrm{kHz}$ did not give the strongest population response in A1 (Fig. $4 A$, right).

Stimulus-specific adaptation impacted animal behavior in a manner consistent with its effects on the amplitude difference between the target and distractor evoked responses in A1. The animals did worse at reporting the side of the target stimulus when the target frequency was adapted, and its evoked response reduced, compared with the condition when the distractor was adapted (Fig. 4B). For 8T4D, performance rose from $59 \pm 2.3 \%$ correct in the target-adapted condition to $74 \pm 2.5 \%$ correct in the distractor-adapted condition ( $\mathrm{M} \pm 95 \% \mathrm{CI}, n=24$ sessions; $p<10^{-5}$, Wilcoxon signed rank test). For 16T8D, performance was significantly below chance when the target was adapted $(42 \pm$ $2.4 \%$ correct), indicating that the animals were consistently reporting the side of the distractor stimulus. Adapting the distractor, however, to make the target deviant, boosted performance to $67 \pm 4.2 \%$ correct $\left(\mathrm{M} \pm 95 \% \mathrm{CI}, n=25\right.$ sessions; $p<10^{-5}$, Wilcoxon signed rank test). Together, our recording and behavioral data imply that rats tend to respond on the side of the stimulus that drives the stronger population response in auditory cortex. Curiously, animals retain this behavioral strategy even when responding to the stronger-amplitude stimulus brings overall less reward than a random response pattern would.

\section{Auditory cortical activity predicts animal task performance}

If evoked response strength in auditory cortex does indeed bias the animals' stimulus selection in the 2-AFC task, then relative target/distractor response strength would be expected to differ between hit and error trials. Specifically, target and distractor responses should show an inverse relationship in amplitude modulation: a higher proportion of stronger target responses should occur on hit trials and a higher proportion of stronger distractor responses should occur on error trials. To assess whether we could see this type of behavioral correlation in A1 activity, we analyzed the eLFP amplitude separately for hit, error, and missed trials (Fig. 5). As predicted, the average target response was stronger on hits than on errors, the differences most apparent when the target was not adapted (solid red curves in the top and bottom; 95\% SEM CIs are within the line thickness). Choice accuracy-related modulation of the distractor response was less consistent, following the expected pattern of stronger distractor response on errors trials only for the adaptation paradigms of the 16T8D pair (Fig. 5D, blue curves). For all stimulus and adaptation conditions, evoked responses on trials that the animals skipped ("missed" trials) were at least as strong as or stronger than performed trial responses.

A convenient way to quantify the target/distractor relationship is to calculate an index of target predominance in the A1 population response $\left(T_{\mathrm{idx}}\right)$, defined as the normalized difference between the target and distractor eLFP amplitudes. $T_{\mathrm{idx}}$ ranges from 1 (target response stronger) to -1 (distractor response stronger). For both $8 \mathrm{~T} 4 \mathrm{D}$ and $16 \mathrm{~T} 8 \mathrm{D}$, the $T_{\mathrm{idx}}$ distributions shown in Figure 6 progressively shift towards 1 as the target response transitions from adapted (left box plots), to non-adapted (central box plots), to deviant (right box plots). For the 16T8D No Adaptation and Target Adapted paradigms, most of the $T_{\mathrm{idx}}$ values are negative, consistent with the $16 \mathrm{kHz}$ target being weaker than the $8 \mathrm{kHz}$ distractor in the eLFP amplitude distributions (Figs. 3C, $4 A$ ).

Comparing the hit, error, and missed trial $T_{\mathrm{idx}}$ distributions confirms the behavioral modulation of A1 activity observed in the grand mean eLFP waveforms of Figure 5. The target response is significantly stronger on hits than on errors in all 16T8D paradigm variations (Fig. $6 B$; Target Adapted: $p=0.02$; No Adaptation: $p<10^{-7}$; Distractor Adapted: $p<10^{-10}$, Wilcoxon rank sum test) as well as in the No Adaptation paradigm of 8T4D (Fig. $6 A ; p<10^{-4}$, Wilcoxon rank sum test). The $T_{\mathrm{idx}}$ is also significantly higher on misses compared with errors for two of the 16T8D paradigms (Fig. 6B; Target Adapted: $p=0.120$; No Adaptation: $p=0.019$; Distractor Adapted: $p<10^{-8}$; Wilcoxon rank sum test). For $8 \mathrm{~T} 4 \mathrm{D}$, the opposite pattern holds true: $T_{\mathrm{idx}}$ is lower on missed trials than on error trials (Fig. 6A; Target Adapted: $p<10^{-6}$; No Adaptation: $p=0.006$; Distractor Adapted: $p=$ 0.003; Wilcoxon rank sum test). There is no significant difference in hit and error $T_{\mathrm{idx}}$ distributions in the two $8 \mathrm{~T} 4 \mathrm{D}$ adaptation paradigms (Fig. $6 A$; Target Adapted: $p=0.07$; Distractor Adapted: $p=0.19$; Wilcoxon rank sum test).

Given that rats are biased to select the stimulus that evokes the stronger response in $\mathrm{A} 1$, we reasoned that a close correlation should be observed between $\mathrm{T}_{\mathrm{idx}}$ and behavioral performance. Plotting performance in the six different stimulus/adaptation paradigms as a function of median hemisphere $T_{\mathrm{idx}}$ for recordings made during the corresponding behavioral sessions gave a linear fit that explained the variance in the data with $r^{2}=0.699$ $\left(p<10^{-9}\right.$; Fig. $\left.7 A\right)$. The same was observed in each subject individually: the higher the median hemisphere $T_{i \mathrm{dx}}$ in a given behavioral session of any paradigm, the better the performance. 

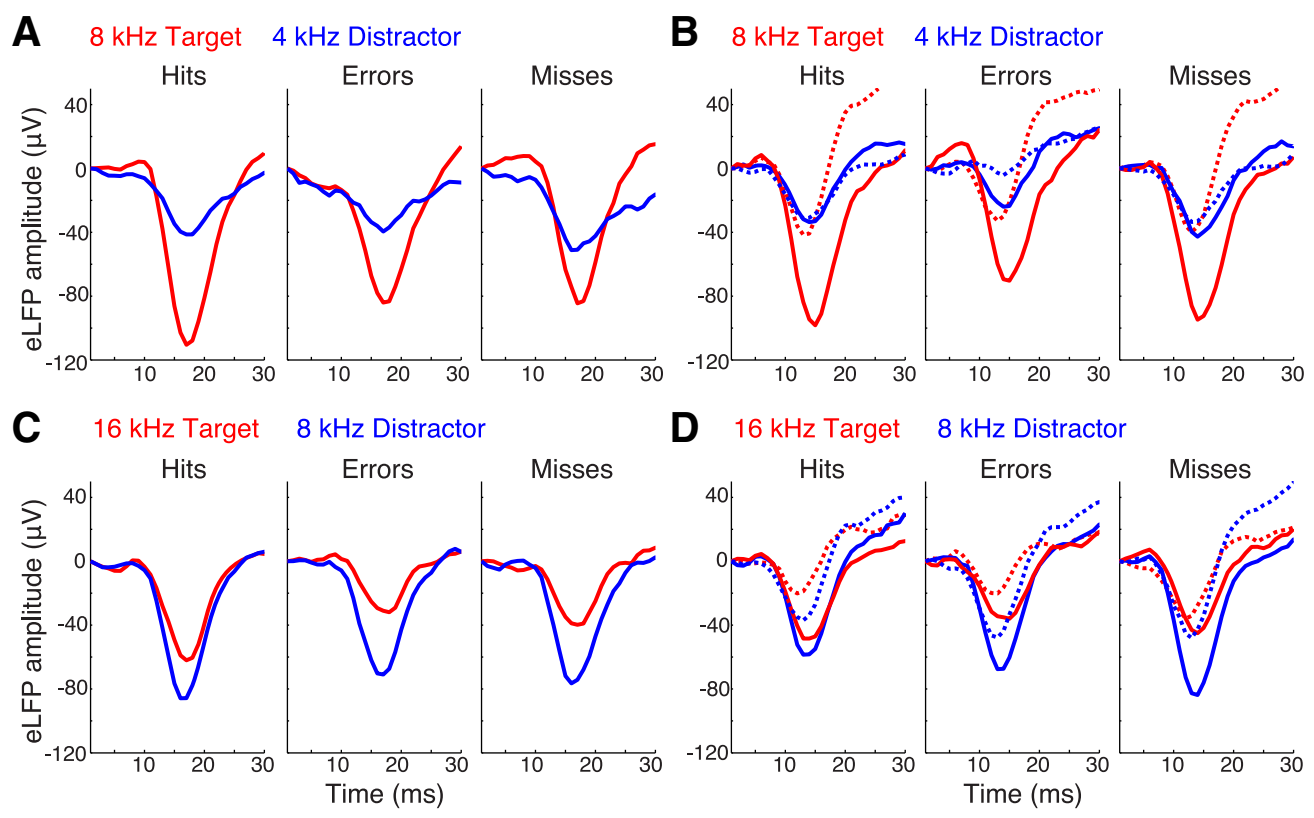

D $16 \mathrm{kHz}$ Target

$8 \mathrm{kHz}$ Distractor

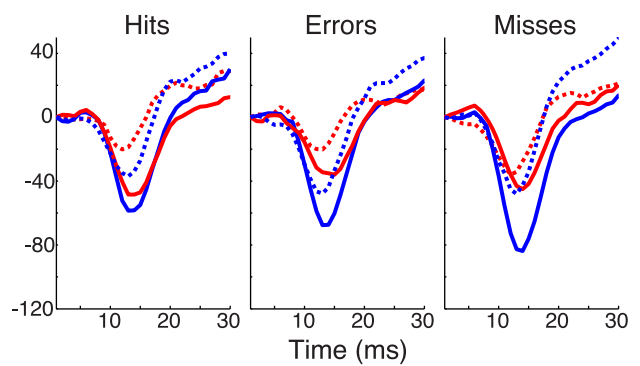

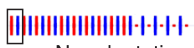

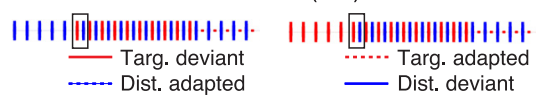

Figure 5. Tone-evoked LFP response strength in A1 varies with behavioral response category. $\boldsymbol{A}$, Auditory cortical responses to 8T4D in the No Adaptation paradigm, and ( $\boldsymbol{B}$ ) the Target Adapted and Distractor Adapted paradigms. C, Auditory cortical responses to 16T8D in the No Adaptation paradigm, and (D) the Target Adapted and Distractor Adapted paradigms. Panels show grand mean eLFP waveforms for the first target (red) and first distractor (blue) tones of the interleaved sequence in the non-adapted, adapted, and deviant conditions, averaged separately for hit, error, and missed (no response) trials across all animals, recording sessions, and channels ( $n=3$ animals, 5 implants, 31 sessions). Ninety-five percent SEM Cls are within the line thickness. The target response is stronger on hit trials than on error trials for all paradigms except 16T8D Target adapted. Across all stimulus and adaptation conditions, missed trial eLFP responses are at least as strong as or stronger than performed trial responses.

Repeated-measures correlation analysis (rmcorr; Bakdash and Marusich, 2017), a method that assays each subject's data for an intra-individual relationship and then finds the common association across individuals by removing intersubject variance, revealed a strong positive relationship between session $T_{\mathrm{idx}}$ and performance (Fig. 7B; $r_{r \mathrm{~m}}=0.68 ; 0.60-0.75$ CI95; $p<10^{-26}$ ). Thus, in our paradigm, tone-evoked activity in Al can serve as an excellent predictor of the animal's stimulus choice, and more generally, as an indicator of perceived stimulus saliency.

\section{Discussion}

To explore the relationship between stimulus representation in A1 and auditory decision-making, we established a novel 2-AFC task in rats modeling the cocktail party problem. Animals were required to report the location of the higher frequency within two interleaved pure-tone sequences. We found that rats tended to report the location of the stimulus driving the stronger eLFP responses in A1, regardless of whether it was the target. Behavioral performance could be improved by reducing distractorevoked activity through SSA. In fact, performance could be shifted either above or below chance, depending on which frequency, distractor or target, was adapted. A linear relationship between eLFP amplitude and behavior was found: the stronger the target population response relative to the distractor, the better the performance. Moreover, Al responses varied with task engagement and with behavioral choice accuracy. Below, we first explore the bottom-up factors affecting stimulus saliency and then go on to consider the possible origins and implications of behaviorally-related differences in A1 activity.

\section{Pure tone frequency and perceptual saliency}

The striking $24 \%$ difference in rats' performance for $8 \mathrm{~T} 4 \mathrm{D}$ compared with $16 \mathrm{~T} 8 \mathrm{D}$ raised questions about the relative perceptual saliency of the target and distractor frequencies in these two stimulus combinations. Sprague Dawley and other rat strains have the lowest hearing threshold at $8 \mathrm{kHz}$, with slightly higher thresholds for 4 and $16 \mathrm{kHz}$. Although all stimuli in our task were presented at the same SPL $(60 \mathrm{~dB})$, their sensation level (SL) relative to the animals' absolute hearing threshold was different, with $8 \mathrm{kHz}$ exceeding $4 \mathrm{kHz}$ by $\sim 10 \mathrm{~dB}$ SPL and exceeding $16 \mathrm{kHz}$ by $\sim 3 \mathrm{~dB}$ SPL (Kelly and Masterton, 1977; Heffner and Heffner, 1985; Heffner et al., 1994). The difference in saliency likely made the task of responding to $8 \mathrm{kHz}$ as the target in the $8 \mathrm{~T} 4 \mathrm{D}$ pairing easier than suppressing responses to $8 \mathrm{kHz}$ as the distractor in the $16 \mathrm{~T} 8 \mathrm{D}$ pairing.

The psychophysically-derived hearing curves have an identified neural correlate. Single-neuron response thresholds at their CFs in rat A1 closely match animals' SL at those frequencies (Sally and Kelly, 1988; Doan and Saunders, 1999). Tones to which rats are most sensitive $(8-40 \mathrm{kHz})$ not only evoke A1 responses at the softest levels, they are disproportionately represented on the A1 tonotopic map (Sally and Kelly, 1988; Rutkowski et al., 2003; Kalatsky et al., 2005). Although the majority of multiunits sampled by our arrays had CFs $>16 \mathrm{kHz}$, the strongest eLFPs were driven by $8 \mathrm{kHz}$ (Fig. $3 \mathrm{C}$ ). These findings are consistent with earlier work showing that although most of the rat A1 tonotopic map has CFs $>8 \mathrm{kHz}$, when tones are presented at $60 \mathrm{~dB} \mathrm{SPL}$, a larger proportion of the map becomes activated in response to 8 $\mathrm{kHz}$ than to either 16 or $4 \mathrm{kHz}$ (Polley et al., 2007).

\section{Behavioral relevance and A1 stimulus representation}

Because our animals were exposed to the $8 \mathrm{kHz}$ stimulus twice as often as to either of the other frequencies, a concern would be that the strong predominance of the $8 \mathrm{kHz}$ response in $\mathrm{A} 1$ population activity (Fig. $3 C$ ) was an artifact of behavioral training. The tono- 
A $8 \mathrm{kHz}$ Target $4 \mathrm{kHz}$ Distractor

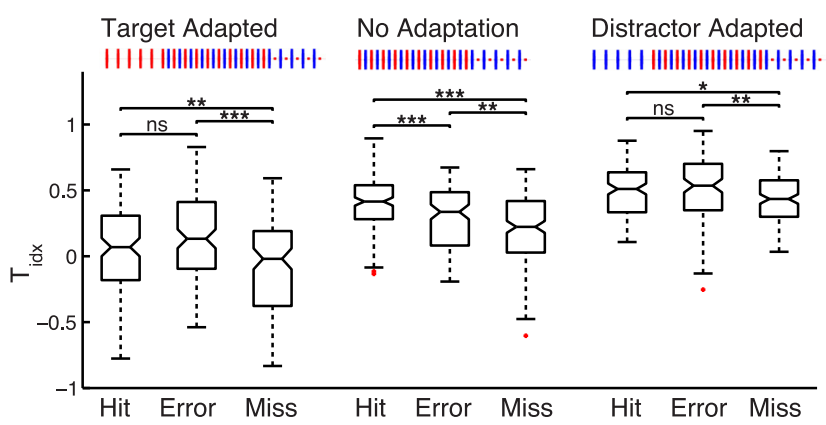

B $16 \mathrm{kHz}$ Target $8 \mathrm{kHz}$ Distractor

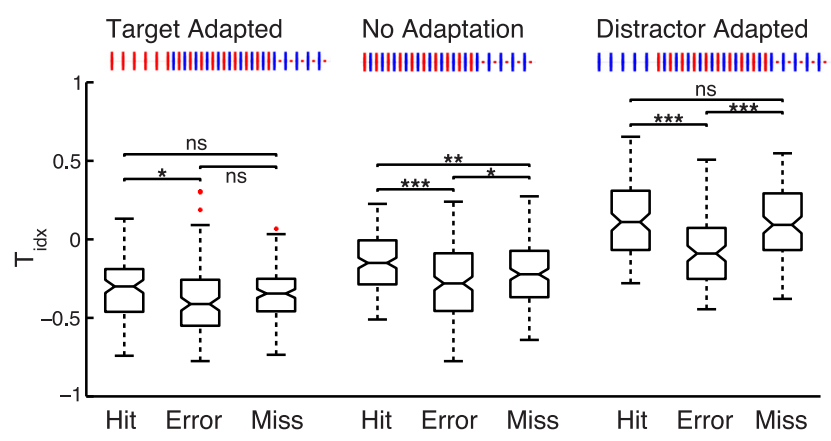

Figure 6. Target-driven activity predominates in rat A1 on correctly-performed trials. A, Distributions of the $T_{\mathrm{idx} x}$ a measure of the relative strength of the target and distractor eLFP responses, for 8T4D and (B) 16T8D. Each data point corresponds to a $T_{\text {idx }}$ value for one stimulusresponsive channel in one recording session (or several consecutive sessions; see Materials and Methods). More positive values indicate a stronger target response. The target index is significantly higher on hit trials compared with error trials in the No Adaptation paradigm for $8 \mathrm{THD}(A)$ and in all paradigm types for $16 \mathrm{~T} 8 \mathrm{D}$ ( $\boldsymbol{B}$; Wilcoxon signed rank test; $\left.{ }^{*} p<0.05,{ }^{* * *} p<0.001\right)$. The missed trial $T_{\text {idx }}$ distributions show opposite trends for the two frequency combinations, shifting in favor of the distractor (more negative) for 8T4D and in favor of the target (more positive) for 16T8D (Wilcoxon signed rank test; ${ }^{*} p<0.05,{ }^{* *} p<0.01,{ }^{* * *} p<0.001$ ). ns, not significant.

A

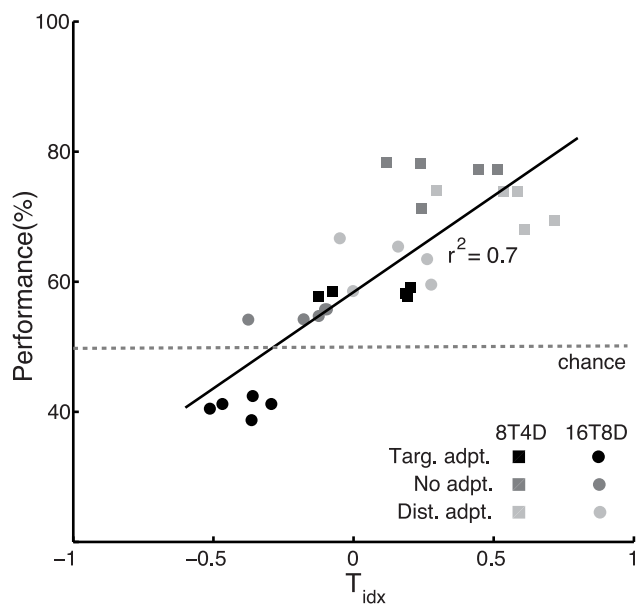

topic maps of Sprague Dawley auditory cortex are not static but expand at the frequency range associated with reward (Polley et al., 2006). Importantly, these changes are not caused by overexposure to the task stimuli, because the exact same stimulation protocol in rats trained to recognize tone loudness instead of frequency does not modify tonotopy. Moreover, in the mouse, prolonged stimulus exposure actually leads to suppression of pyramidal cell tone-driven responses coupled with an increase of interneuron activity. When mice start using the stimuli to perform a behavioral task, however, the opposite modulation of the two cell classes occurs (Kato et al., 2015). Similarly, task relevance is necessary for frequency-specific tonotopic expansion in primates. The size of the area in A1 dedicated to the target frequency correlates with the level of the monkeys' perceptual acuity (Recanzone et al., 1993). Analogously, in rats, the degree of rewarded-frequency expansion predicts animals' performance (Rutkowski and Weinberger, 2005).

Although in our paradigm, $8 \mathrm{kHz}$ was presented to the animals on every trial in combination with either 4 or $16 \mathrm{kHz}$, it was associated with reward in only half. Thus, any reward-associated frequency expansion should have affected $16 \mathrm{kHz}$ to the same extent, had the animals' performance been even for the two frequency pairings from the beginning of training. The initial SL imbalance between the stimuli, however, along with the difference in performance, may have conferred an advantage onto 8 $\mathrm{kHz}$, expanding its cortical representation to a greater degree.

\section{Auditory adaptation and sensory discrimination}

One of the key outcomes of our experiments is that better neural discriminability is associated with better perceptual discriminability. A powerful bottom-up way to improve discriminability of the stimuli in our task turned out to be SSA, the process by which neural response amplitude adjusts to reflect information content of the input: whereas rare stimuli evoke stronger activity, responses to common stimuli are reduced (Wark et al., 2007). Previous work has shown that rats' frequency discrimination thresholds for pure tones improve twofold when one of the com-

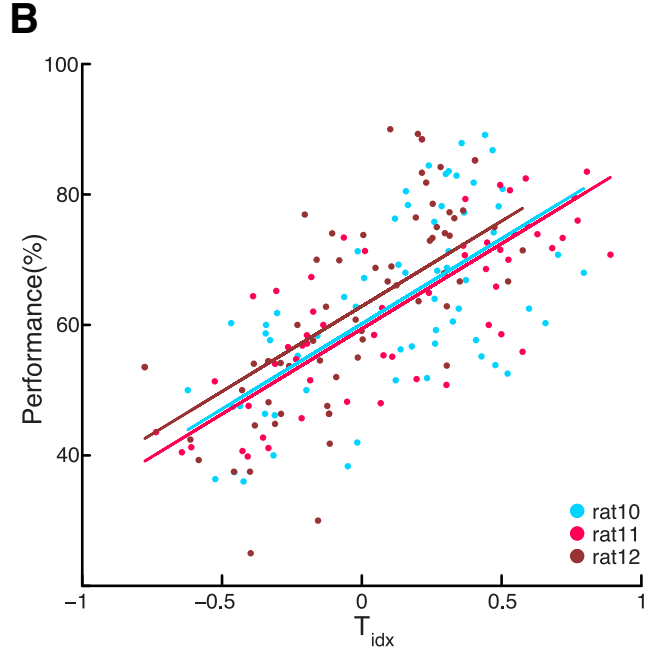

Figure 7. Target predominance index predicts animal performance by paradigm type and session-by-session. $\boldsymbol{A}, \mathrm{A}$ close correlation $\left(r^{2}=0.699, p<10^{-9}\right)$ is observed between median hemisphere $T_{\text {idx }}$ and median performance of each paradigm type: the stronger the A1 population response to the target relative to the distractor, the better the animals are at discriminating and localizing the target stimulus. Each data point plots the median $T_{\mathrm{idx}}$ of all responsive channels in a given implanted array versus median animal performance in all sessions of a given paradigm type recorded with that array (8T4D, squares; 16T8D, circles; with different greyscale shades corresponding to the different adaptation conditions). $\boldsymbol{B}$, The same relationship holds for single-session performance in each rat. Repeated measures correlation analysis reveals a strong positive within-subject association between median hemisphere single-session $T_{\mathrm{idx}}$ and individual session performance $\left(r_{\mathrm{rm}}=0.68 ; 0.60-0.75\left(195 ; p<10^{-26}\right)\right.$. All paradigm types are included for each rat. Regression lines show the intrasubject association between $T_{\mathrm{idx}}$ and performance that all subjects share (a common regression slope). 
pared tones is adapted (Syka et al., 1996; Talwar and Gerstein, 1998). Similarly, adaptation improves rats' spatial discrimination of vibrotactile stimuli (Ollerenshaw et al., 2014). In our paradigm, rats tended to report the location of the most-salient stimulus and performed near chance when the saliency of the interleaved frequencies was similar (as in 16T8D; Fig. 3D). By varying whether the target frequency was the deviant or the adapted sound, we could shift the median target index of the A1 population from positive (target-dominant) to negative (distractor-dominant; Fig. $6 B$ ), which in turn shifted performance either above or below chance (Fig. $4 B$ ).

While few studies before ours have addressed the effects of cortical SSA on auditory perception (although extensive work has been done in humans with a related phenomenon, mismatch negativity; Tiitinen et al., 1994; Winkler et al., 2009), classic studies in brainstem circuits have demonstrated that auditory adaptation can profoundly influence behavior. In prepulse inhibition, an acoustic startle response elicited by a very loud sound can be diminished or abolished by presenting a moderately-loud tone $10-100 \mathrm{~ms}$ earlier. The more space a particular frequency representation takes up in the inferior colliculus, the more that frequency will inhibit the animal's startle response (Carlson and Willott, 1996). It can be inferred that the strength of the neural response a particular stimulus evokes sets its capacity for dampening the saliency of stimuli that follow. Having found that 8 $\mathrm{kHz}$, the frequency driving the strongest $\mathrm{A} 1$ population response (Fig. $3 C$ ), also exhibits the widest dynamic range of SSA-induced response amplitude modulation (Fig. $4 A$ ), we suggest that the changes in animal performance in the different adaptation paradigms (Fig. 4B) were primarily caused by dampened $8 \mathrm{kHz}$ drive.

One caveat to the above interpretation is the existence of SSA in subcortical auditory structures, which plays a major role in sound localization and could have caused the SSA-related behavioral changes observed. The primary auditory cortex is considered to be the first lemniscal station of the ascending auditory pathway to exhibit strong SSA (Khouri and Nelken, 2015), whereas in subcortical auditory structures, SSA is particularly prominent in the non-lemniscal parts (Anderson et al., 2009; Antunes et al., 2010; Bäuerle et al., 2011), which are subject to extensive corticofugal feedback (Terreros and Delano, 2015). Inactivating the auditory cortex silences thalamic SSA (Bäuerle et al., 2011), indicating that SSA is projected back at least to the thalamus and probably even to the inferior colliculus. Thus, the observed effects of SSA on behavior are likely of cortical origin, although inactivation studies would need to be done to make a definitive claim.

\section{A1 activity in the passive and engaged behavioral states}

A key advantage of 2-AFC paradigms over go/no-go paradigms is the distinction of error trials from trials in which the animal does not respond because of low motivation. Recording neural activity during these missed trials allowed us to address an ambiguity in the field: how A1 responses differ between the passive and active behavioral states. In primate auditory cortex, task engagement has been shown to give either no activity changes (Hocherman et al., 1976; Gilat and Perlman, 1984; Massoudi et al., 2014) or increased firing rates (Miller et al., 1972; Benson and Hienz, 1978; Scott et al., 2007; Massoudi et al., 2013). In rodents, however, task engagement has traditionally been associated with activity suppression. Barrel cortex responses to brief whisker deflections are weaker when animals are actively whisking as opposed to sitting quietly (Fanselow and Nicolelis, 1999; Crochet and Petersen, 2006; Ferezou et al., 2006). In rat auditory cortex, activity is sup- pressed when animals transition from passive listening to active auditory localization (Otazu et al., 2009), whereas in gerbil A1, spontaneous activity decreases during task performance (Buran et al., 2014).

In agreement with prior rodent studies, we find evoked responses in the passive state to be stronger than at least one of the performed trial categories in the vast majority of cases (missed trials; Fig. 5). However, the eLFP amplitudes in the other performed trial category (either hits or errors, depending on the paradigm type) often match or exceed the missed trial response. Our results thus support the hypothesis that activity suppression associated with task engagement reflects a general state of alertness onto which other gain-modulatory mechanisms may be overlaid (Otazu et al., 2009) and highlights the importance of distinguishing between correct and incorrect trials rather than grouping them into a general "performed" category.

\section{Choice accuracy-related differences in A1 activity}

Our data reveals a clear relationship between the bottom-up saliency of an auditory stimulus and rats' tendency to select it over others in the cocktail party setting. Whether and how the animals' internal state affects Al activity is less clear. For example, $T_{\mathrm{idx}}$ is higher on hit trials compared to error trials in all three 16T8D paradigms, but only in the No Adaptation paradigm of 8T4D (Fig. 6). Examining the modulation of target and distractor eLFP waveforms that underlie the shifts in $T_{\mathrm{idx}}$, we find the target eLFP peak amplitude greater on hits than errors for five of the six paradigms (Fig. 5, red traces). It is tempting to implicate selective attention in this response modulation, as numerous studies have shown that stimulus discriminability in the auditory cortex improves with attention, which boosts gain of target-tuned cells (Atiani et al., 2009; Ahveninen et al., 2011; Lee and Middlebrooks, 2011; Lakatos et al., 2013; Carcea et al., 2017; Downer et al., 2017).

The animals' attention in this task was not controlled, however. Moreover, our analysis concerned just the LFPs evoked by the first two tones of the target-distractor sequence, which the animals had to compare to make a choice. Thus, if the animals' internal state did in fact impact A1 processing, we could only have measured the effects of stimulus expectation before trial onset rather than the effects of an evidence-based sensory decision. Because rats could not predict which stimulus pair would be presented in an upcoming trial, this expectation would be random or biased by the outcome of the previous trial and the overall reward history. Nevertheless, assuming that the expectation of a given frequency would boost A1 responses to it (Voisin et al., 2006; Jaramillo and Zador, 2011), the probability of a correct response would increase on trials in which the expected frequency coincided with the target by chance. Thus, responses with higher-amplitude target eLFPs would be over-represented in the hit trial category.

An alternative interpretation is that trial-by-trial variability in the external signal (input noise) or subcortical processing led to the observed differences in A1 responses, making the target appear more or less salient and a correct response more or less probable. Although our study cannot distinguish between the sources of variability in A1 activity (internal or external noise), our results indicate that the representation of stimulus strength in A1 biases decisions toward the most salient auditory choice.

\section{References}

Ahveninen J, Hämäläinen M, Jääskeläinen IP, Ahlfors SP, Huang S, Lin FH, Raij T, Sams M, Vasios CE, Belliveau JW (2011) Attention-driven audi- 
tory cortex short-term plasticity helps segregate relevant sounds from noise. Proc Natl Acad Sci U S A 108:4182-4187.

Anderson LA, Christianson GB, Linden JF (2009) Stimulus-specific adaptation occurs in the auditory thalamus. J Neurosci 29:7359-7363.

Antunes FM, Nelken I, Covey E, Malmierca MS (2010) Stimulus-specific adaptation in the auditory thalamus of the anesthetized rat. PloS One 5:e14071.

Atiani S, Elhilali M, David SV, Fritz JB, Shamma SA (2009) Task difficulty and performance induce diverse adaptive patterns in gain and shape of primary auditory cortical receptive fields. Neuron 61:467-480.

Bakdash JZ, Marusich LR (2017) Repeated measures correlation. Front Psychol 8:456.

Bäuerle P, von der Behrens W, Kössl M, Gaese BH (2011) Stimulus-specific adaptation in the gerbil primary auditory thalamus is the result of a fast frequency-specific habituation and is regulated by the corticofugal system. J Neurosci 31:9708-9722.

Bendixen A, Schröger E, Winkler I (2009) I heard that coming: event-related potential evidence for stimulus-driven prediction in the auditory system. J Neurosci 29:8447-8451.

Benson DA, Hienz RD (1978) Single-unit activity in the auditory cortex of monkeys selectively attending left vs. right ear stimuli. Brain Res 159:307320.

Bizley JK, Walker KM, Nodal FR, King AJ, Schnupp JW (2013) Auditory cortex represents both pitch judgments and the corresponding acoustic cues. Curr Biol 23:620-625.

Buran BN, von Trapp G, Sanes DH (2014) Behaviorally gated reduction of spontaneous discharge can improve detection thresholds in auditory cortex. J Neurosci 34:4076-4081.

Carcea I, Insanally MN, Froemke RC (2017) Dynamics of auditory cortical activity during behavioural engagement and auditory perception. Nat Commun 8:14412.

Carlson S, Willott JF (1996) The behavioral salience of tones as indicated by prepulse inhibition of the startle response: relationship to hearing loss and central neural plasticity in C57BL/6J mice. Hear Res 99:168-175.

Cherry EC (1953) Some experiments on the recognition of speech with one and two ears. J Acoust Soc Am 25:975-979.

Crochet S, Petersen CC (2006) Correlating whisker behavior with membrane potential in barrel cortex of awake mice. Nat Neurosci 9:608-610.

Deneux T, Kempf A, Daret A, Ponsot E, Bathellier B (2016) Temporal asymmetries in auditory coding and perception reflect multi-layered nonlinearities. Nat Commun 7:12682.

Doan DE, Saunders JC (1999) Sensitivity to simulated directional sound motion in the rat primary auditory cortex. J Neurophysiol 81:2075-2087.

Dong C, Qin L, Liu Y, Zhang X, Sato Y (2011) Neural responses in the primary auditory cortex of freely behaving cats while discriminating fast and slow click-trains. PloS One 6:e25895.

Downer JD, Rapone B, Verhein J, O'Connor KN, Sutter ML (2017) Feature selective attention adaptively shifts noise correlations in primary auditory cortex. J Neurosci 37:5378-5392.

Fanselow EE, Nicolelis MA (1999) Behavioral modulation of tactile responses in the rat somatosensory system. J Neurosci 19:7603-7616.

Ferezou I, Bolea S, Petersen CC (2006) Visualizing the cortical representation of whisker touch: voltage-sensitive dye imaging in freely moving mice. Neuron 50:617-629.

Fritz J, Shamma S, Elhilali M, Klein D (2003) Rapid task-related plasticity of spectrotemporal receptive fields in primary auditory cortex. Nat Neurosci 6:1216-1223.

Gaucher Q, Edeline JM, Gourévitch B (2012) How different are the local field potentials and spiking activities? Insights from multi-electrodes arrays. J Physiol Paris 106:93-103.

Gilat E, Perlman I (1984) Single unit activity in the auditory cortex and the medial geniculate body of the rhesus monkey: behavioral modulation. Brain Res 324:323-333.

Heffner HE, Heffner RS (1985) Hearing in two cricetid rodents: wood rat (Neotoma floridana) and grasshopper mouse (Onychomys leucogaster). J Comp Psychol 99:275-288.

Heffner HE, Heffner RS, Contos C, Ott T (1994) Audiogram of the hooded Norway rat. Hear Res 73:244-247.

Hocherman S, Benson DA, Goldstein MH Jr, Heffner HE, Hienz RD (1976) Evoked unit activity in auditory cortex of monkeys performing a selective attention task. Brain Res 117:51-68.
Hromádka T, Deweese MR, Zador AM (2008) Sparse representation of sounds in the unanesthetized auditory cortex. PLoS Biol 6:e16.

Jaramillo S, Zador AM (2011) The auditory cortex mediates the perceptual effects of acoustic temporal expectation. Nat Neurosci 14:246-251.

Kalatsky VA, Polley DB, Merzenich MM, Schreiner CE, Stryker MP (2005) Fine functional organization of auditory cortex revealed by Fourier optical imaging. Proc Natl Acad Sci U S A 102:13325-13330.

Kato HK, Gillet SN, Isaacson JS (2015) Flexible sensory representations in auditory cortex driven by behavioral relevance. Neuron 88:1027-1039.

Kayser C, Petkov CI, Lippert M, Logothetis NK (2005) Mechanisms for allocating auditory attention: an auditory saliency map. Curr Biol 15:1943-1947.

Kelly JB, Masterton B (1977) Auditory sensitivity of the albino rat. J Comp Physiol Psychol 91:930-936.

Khouri L, Nelken I (2015) Detecting the unexpected. Curr Opin Neurobiol 35:142-147.

Lakatos P, Musacchia G, O'Connel MN, Falchier AY, Javitt DC, Schroeder CE (2013) The spectrotemporal filter mechanism of auditory selective attention. Neuron 77:750-761.

Lee CC, Middlebrooks JC (2011) Auditory cortex spatial sensitivity sharpens during task performance. Nat Neurosci 14:108-114.

Lemus L, Hernández A, Romo R (2009) Neural codes for perceptual discrimination of acoustic flutter in the primate auditory cortex. Proc Natl Acad Sci U S A 106:9471-9476.

Massoudi R, Van Wanrooij MM, Van Wetter SM, Versnel H, Van Opstal AJ (2013) Stable bottom-up processing during dynamic top-down modulations in monkey auditory cortex. Eur J Neurosci 37:1830-1842.

Massoudi R, Van Wanrooij MM, Van Wetter SM, Versnel H, Van Opstal AJ (2014) Task-related preparatory modulations multiply with acoustic processing in monkey auditory cortex. Eur J Neurosci 39:1538-1550.

Mayrhofer JM, Skreb V, von der Behrens W, Musall S, Weber B, Haiss F (2013) Novel two-alternative forced choice paradigm for bilateral vibrotactile whisker frequency discrimination in head-fixed mice and rats. J Neurophysiol 109:273-284.

Miller JM, Sutton D, Pfingst B, Ryan A, Beaton R, Gourevitch G (1972) Single cell activity in the auditory cortex of rhesus monkeys: behavioral dependency. Science 177:449-451.

Musall S, von der Behrens W, Mayrhofer JM, Weber B, Helmchen F, Haiss F (2014) Tactile frequency discrimination is enhanced by circumventing neocortical adaptation. Nat Neurosci 17:1567-1573.

Näätänen R, Schröger E, Karakas S, Tervaniemi M, Paavilainen P (1993) Development of a memory trace for a complex sound in the human brain. Neuroreport 4:503-506.

Nelken I (2004) Processing of complex stimuli and natural scenes in the auditory cortex. Curr Opin Neurobiol 14:474-480.

Niwa M, Johnson JS, O'Connor KN, Sutter ML (2012) Activity related to perceptual judgment and action in primary auditory cortex. J Neurosci 32:3193-3210.

Noda T, Kanzaki R, Takahashi H (2013) Stimulus phase locking of cortical oscillation for auditory stream segregation in rats. PloS One 8:e83544.

Ollerenshaw DR, Zheng HJ, Millard DC, Wang Q, Stanley GB (2014) The adaptive trade-off between detection and discrimination in cortical representations and behavior. Neuron 81:1152-1164.

Otazu GH, Tai LH, Yang Y, Zador AM (2009) Engaging in an auditory task suppresses responses in auditory cortex. Nat Neurosci 12:646-654.

Polley DB, Steinberg EE, Merzenich MM (2006) Perceptual learning directs auditory cortical map reorganization through top-down influences. J Neurosci 26:4970-4982.

Polley DB, Read HL, Storace DA, Merzenich MM (2007) Multiparametric auditory receptive field organization across five cortical fields in the albino rat. J Neurophysiol 97:3621-3638.

Profant O, Burianová J, Syka J (2013) The response properties of neurons in different fields of the auditory cortex in the rat. Hear Res 296:51-59.

Recanzone GH, Schreiner CE, Merzenich MM (1993) Plasticity in the frequency representation of primary auditory cortex following discrimination training in adult owl monkeys. J Neurosci 13:87-103.

Rodgers CC, DeWeese MR (2014) Neural correlates of task switching in prefrontal cortex and primary auditory cortex in a novel stimulus selection task for rodents. Neuron 82:1157-1170.

Rothschild G, Nelken I, Mizrahi A (2010) Functional organization and population dynamics in the mouse primary auditory cortex. Nat Neurosci 13:353-360. 
Rutkowski RG, Weinberger NM (2005) Encoding of learned importance of sound by magnitude of representational area in primary auditory cortex. Proc Natl Acad Sci U S A 102:13664-13669.

Rutkowski RG, Miasnikov AA, Weinberger NM (2003) Characterisation of multiple physiological fields within the anatomical core of rat auditory cortex. Hear Res 181:116-130.

Sally SL, Kelly JB (1988) Organization of auditory cortex in the albino rat: sound frequency. J Neurophysiol 59:1627-1638.

Schwarz C, Hentschke H, Butovas S, Haiss F, Stüttgen MC, Gerdjikov TV, Bergner CG, Waiblinger C (2010) The head-fixed behaving rat: procedures and pitfalls. Somatosens Mot Res 27:131-148.

Scott BH, Malone BJ, Semple MN (2007) Effect of behavioral context on representation of a spatial cue in core auditory cortex of awake macaques. J Neurosci 27:6489-6499.

Selezneva E, Scheich H, Brosch M (2006) Dual time scales for categorical decision making in auditory cortex. Curr Biol 16:2428-2433.

Selezneva E, Oshurkova E, Scheich H, Brosch M (2017) Category-specific neuronal activity in left and right auditory cortex and in medial geniculate body of monkeys. PloS One 12:e0186556.

Syka J, Rybalko N, Brozek G, Jilek M (1996) Auditory frequency and intensity discrimination in pigmented rats. Hear Res 100:107-113.

Szymanski FD, Garcia-Lazaro JA, Schnupp JW (2009) Current source density profiles of stimulus-specific adaptation in rat auditory cortex. J Neurophysiol 102:1483-1490.

Talwar SK, Gerstein GL (1998) Auditory frequency discrimination in the white rat. Hear Res 126:135-150.
Terreros G, Delano PH (2015) Corticofugal modulation of peripheral auditory responses. Front Syst Neurosci 9:134.

Tervaniemi M, Maury S, Näätänen R (1994) Neural representations of abstract stimulus features in the human brain as reflected by the mismatch negativity. Neuroreport 5:844-846.

Tiitinen H, May P, Reinikainen K, Nääänen R (1994) Attentive novelty detection in humans is governed by pre-attentive sensory memory. $\mathrm{Na}-$ ture 372:90-92.

Ulanovsky N, Las L, Farkas D, Nelken I (2004) Multiple time scales of adaptation in auditory cortex neurons. J Neurosci 24:10440-10453.

Voisin J, Bidet-Caulet A, Bertrand O, Fonlupt P (2006) Listening in silence activates auditory areas: a functional magnetic resonance imaging study. J Neurosci 26:273-278.

von der Behrens W, Bäuerle P, Kössl M, Gaese BH (2009) Correlating stimulus-specific adaptation of cortical neurons and local field potentials in the awake rat. J Neurosci 29:13837-13849.

Wark B, Lundstrom BN, Fairhall A (2007) Sensory adaptation. Curr Opin Neurobiol 17:423-429.

Wiegrebe L, Winter IM (2001) Temporal representation of iterated rippled noise as a function of delay and sound level in the ventral cochlear nucleus. J Neurophysiol 85:1206-1219.

Winkler I, Denham SL, Nelken I (2009) Modeling the auditory scene: predictive regularity representations and perceptual objects. Trends Cogn Sci 13:532-540

Yao JD, Bremen P, Middlebrooks JC (2013) Rat primary auditory cortex is tuned exclusively to the contralateral hemifield. J Neurophysiol 110:2140-2151. 